\title{
In search of stars: network formation among heterogeneous agents
}

Citation for published version (APA):

Riedl, A. M., Goeree, J. K., \& Ule, A. (2009). In search of stars: network formation among heterogeneous agents. Games and Economic Behavior, 67(2), 445-466. https://doi.org/10.1016/j.geb.2008.12.005

Document status and date:

Published: 01/01/2009

DOI:

10.1016/j.geb.2008.12.005

Document Version:

Publisher's PDF, also known as Version of record

Document license:

Taverne

\section{Please check the document version of this publication:}

- A submitted manuscript is the version of the article upon submission and before peer-review. There can be important differences between the submitted version and the official published version of record.

People interested in the research are advised to contact the author for the final version of the publication, or visit the DOI to the publisher's website.

- The final author version and the galley proof are versions of the publication after peer review.

- The final published version features the final layout of the paper including the volume, issue and page numbers.

Link to publication

\footnotetext{
General rights rights.

- You may freely distribute the URL identifying the publication in the public portal. please follow below link for the End User Agreement:

www.umlib.nl/taverne-license

Take down policy

If you believe that this document breaches copyright please contact us at:

repository@maastrichtuniversity.nl

providing details and we will investigate your claim.
}

Copyright and moral rights for the publications made accessible in the public portal are retained by the authors and/or other copyright owners and it is a condition of accessing publications that users recognise and abide by the legal requirements associated with these

- Users may download and print one copy of any publication from the public portal for the purpose of private study or research.

- You may not further distribute the material or use it for any profit-making activity or commercial gain

If the publication is distributed under the terms of Article $25 \mathrm{fa}$ of the Dutch Copyright Act, indicated by the "Taverne" license above, 


\title{
In search of stars: Network formation among heterogeneous agents
}

\author{
Jacob K. Goeree ${ }^{\mathrm{a}}$, Arno Riedl ${ }^{\mathrm{b}}$, Aljaž Ule ${ }^{\mathrm{c}, *}$ \\ a Division of the Humanities and Social Sciences, California Institute of Technology, Mail code 228-77, Pasadena, CA 91125, USA \\ ${ }^{\mathrm{b}}$ Department of Economics, Faculty of Economics and Business Administration, Maastricht University, PO Box 616, 6200 MD, Maastricht, The Netherlands \\ c CREED, Faculty of Economics and Business, University of Amsterdam, Roetersstraat 11, 1018 WB, Amsterdam, The Netherlands
}

\section{A R T I C L E I N F O}

\section{Article history:}

Received 24 July 2006

Available online 31 December 2008

\section{JEL classification:}

$\mathrm{C} 72$

C92

D82

D85

\section{Keywords:}

Network formation

Experiment

Heterogeneity

Private information

\begin{abstract}
A B S T R A C T
This paper reports results from a laboratory experiment on network formation among heterogeneous agents. The experimental design extends the Bala-Goyal [Bala, V., Goyal, S., 2000. A non-cooperative model of network formation, Econometrica 68, 1131-1230] model of network formation with decay and two-way flow of benefits by introducing agents with lower linking costs or higher benefits to others. Furthermore, agents' types may be common knowledge or private information. In all treatments, the (efficient) equilibrium network has a "star" structure. While equilibrium predictions fail completely with homogeneous agents, star networks frequently occur with heterogeneous agents. Stars are not born but rather develop: with a high-value agent, the network's centrality, stability, and efficiency all increase over time. A structural econometric model based on best response dynamics and other-regarding preferences is used to analyze individual linking behavior. Maximum-likelihood estimates of the underlying structural parameters, obtained by pooling data from several treatments, allow us to explain the main treatment effects.
\end{abstract}

(c) 2008 Elsevier Inc. All rights reserved.

\section{Introduction}

Many social, economic, and information networks exhibit uneven, hierarchical structures. The Internet, for example, consists of relatively few web-sites with a huge number of links and a preponderance of web-pages with only a few links. ${ }^{1}$ Co-author networks typically display a few well-connected researchers collaborating with many others. ${ }^{2}$ Finally, casual observation suggests that ongoing social relations require the presence of relatively few active organizers who are central to a large network of friends or colleagues.

Several explanations have been put forth to explain the emergence of such hierarchical structures. Barabási and Albert (1999) demonstrate that hub-like structures can result from a simple dynamic process where the probability that a new agent entering the network connects with an existing agent is proportional to the number of links the existing agent has. They do not, however, give a rationale for such linking behavior. Jackson and Rogers (2007) consider a model where entering agents perform local searches in the neighborhoods of agents they randomly meet. They show that the resulting model is

\footnotetext{
Wh gratefully acknowledge financial support from the National Science Foundation (0551014), the Gordon and Betty Moore Foundation, and the Dutch National Science Foundation NWO (VICI 453.03.606 and VENI 451-07-031).

* Corresponding author. Fax: +31 205255283.

E-mail addresses: jkg@hss.caltech.edu (J.K. Goeree), a.riedl@algec.unimaas.nl (A. Riedl), a.ule@uva.nl (A. Ule).

1 See, e.g., Barabási (2002).

2 See, e.g., Newman (2004) and Goyal et al. (2006).
} 
capable of reproducing many characteristics of socially generated networks. Both papers focus on large networks with agents that have imperfect knowledge about the prevailing network structure. ${ }^{3}$

In contrast, Bala and Goyal (2000) study a game-theoretic model where agents possess complete information about the network, an assumption that is probably more realistic in small networks with relatively few agents. Their approach is based on the idea that a link between two agents is established whenever at least one of them is willing to pay the cost. ${ }^{4}$ Bala and Goyal show that extremely uneven or hierarchical networks can arise in equilibrium if benefits can be accessed through the network regardless of who sponsors its links (two-way flow of benefits). Indeed, for a wide range of parameter values the predicted equilibrium network has a "star"-like structure with a single agent (the center) being directly connected to all other (peripheral) agents. ${ }^{5}$ Stars are predicted to occur even though agents are symmetric with identical incentives and opportunities.

Network formation is hard to investigate in the field because of many potentially confounding factors, e.g., possible asymmetries in agents' information or motivations, imperfect knowledge about linking opportunities or existing network structures, etc. A valuable alternative is provided by controlled laboratory experiments, an approach used by Falk and Kosfeld (2005) to study network formation among homogeneous agents. They find that linking behavior does not converge in settings where the unique strict Nash equilibrium network has a star-like structure ${ }^{6}$ and argue that strategic asymmetry and payoff asymmetry account for the failure of Nash predictions. ${ }^{7}$ These negative results are interesting in that they highlight the difficulties in forming star-like networks even in small groups of (four) agents who possess complete information about others' types and the prevailing network structure.

As the aforementioned examples indicate, however, star-like networks do emerge in the real-world. One obvious difference between the experimental setup and the real-life examples is the assumed symmetry across agents. Casual observation suggests that in practice, individual differences may play an important role in network formation. For instance, some people are better "networkers" in that they have a taste for linking to others or lower opportunity costs of maintaining their connections. Likewise, some people possess skills that are more scarce, making them more valuable to others. Individual differences in linking costs or benefits-to-others may resolve some of the strategic asymmetry faced by the agents, e.g., a high-value co-author may be more easily targeted to become the center of a periphery-sponsored star. Furthermore, individual heterogeneity may alleviate payoff asymmetries or make such asymmetries more acceptable. Indeed, the Greek proverb "success has many friends" suggests people have a preference for being connected to highly-rewarded individuals despite the resulting payoff inequalities.

The impact of individual heterogeneity on actual network formation is difficult to address using field data since "linking costs" and "benefits to others" are hard to measure and even harder to vary in a systematic way. The experiments reported in this paper provide a careful evaluation of the effects of asymmetries on linking behavior in a controlled laboratory setting. In addition to a homogeneous environment where all agents have identical linking costs and are of equal value to others, we consider heterogeneous environments where one of the agents has lower linking costs, or a higher value to others, or a combination of two such agents. We consider cases where agents' types are common knowledge and those where their types are private information. The introduction of incomplete information adds realism to the setup and allows us to the study whether not knowing others' types hampers coordination or alleviates mis-coordination due to other-regarding preferences (e.g., envy). For each of the resulting treatments, we provide a complete characterization of the (Bayesian) Nash equilibrium networks and study (i) whether uneven structures emerge, (ii) what network positions different types of agents occupy, (iii) how efficient and stable the observed networks are, and (iv) what factors determine individual linking behavior.

We find that the introduction of different types of agents has a dramatic impact on linking behavior and observed networks. While almost no stars are formed among symmetric agents, they are prevalent in some of the heterogeneous treatments. Recall that in Bala and Goyal's (2000) model of network formation among homogeneous agents, star networks do arise in equilibrium. This theoretical prediction was not confirmed by earlier experimental studies (Falk and Kosfeld, 2005), and we provide insights for why it was not. We show that heterogeneity does not simply resolve coordination problems by introducing 'focal' agents. In some of our treatments with a single 'focal' player, stars do form while in others they do not. Also in a treatment with two high-value agents, stars form even though both agents are equally focal. We develop a theoretic and econometric model based on best-response dynamics and inequality-averse preferences to explain these differences across treatments.

Stars are not born but develop over time: none of the treatments show a significant number of stars in the first five rounds of the experiments, but in several treatments stars are the most prevalent architecture in the final five rounds.

\footnotetext{
3 The resulting network typically contains many different sub-architectures and the distribution of the number of agents' links is approximately "scalefree" (i.e. follows a power law), see, e.g., Barabási and Albert (1999) and Barabási (2002).

4 See Jackson and Wolinsky (1996) for a model where links require both agents' consent. Jackson (2005) provides a recent comprehensive survey of the literature on networks.

5 A similar prediction is true for the symmetric connections model studied by Jackson and Wolinsky (1996).

6 Falk and Kosfeld (2005) include treatments where only the agent that sponsors the link receives the benefits from the link (one-way flow model), see also Callander and Plott (2005). In this case, observed linking behavior does converge to the Nash equilibrium network, which has a "wheel"-type structure. See Corbae and Duffy (2008) for an earlier network experiment and Kosfeld (2004) for a recent survey.

7 Strategic asymmetry reflects the idea that in a completely symmetric setting it may be hard for subjects to coordinate on a very asymmetric outcome such as a star. Likewise, inequality averse subjects may find it hard to accept the payoff asymmetries that occur in star-like networks.
} 
Network centrality displays a strong tendency to rise over time in treatments with a high-value agent. A similar trend is observed for the network's efficiency and its stability. In summary, while star formation is absent initially (and remains absent with symmetric agents), repetition and experience enable subjects to coordinate on these hierarchical structures in some of the heterogeneous treatments.

Incomplete information generally hinders star formation. In treatments with a high-value agent the periphery-sponsored star with this agent in the center is a frequent outcome in both information conditions, and the slight differences across information conditions are not significant. Likewise, in treatments with a low-cost agent, incomplete information raises the occurrence of stars with the low-cost agent at the center slightly, but again, this difference is not significant. Finally, when both a low-cost and a high-value agent are present, incomplete information clearly aggravates the coordination problem subjects face: fewer stars are formed and not all of them are periphery-sponsored stars with the high-value agent at the center. In contrast, the abundance of stars observed for the complete information case are all of this type. The differences between the information conditions are significant in this case.

We also test how heterogeneity affects individual behavior. We estimate a structural econometric model based on bestresponse dynamics and inequality-averse preferences to explain agents' linking decisions. In this model, agents experience envy (guilt) when others' net payoffs from the formed network are higher (lower). ${ }^{8}$ In most treatments, envy (more than guilt) plays a significant role in individual decision making. In particular, the maximum-likelihood estimates of the envy parameter (obtained by pooling data from several treatments) explain why star formation is much more likely in treatments with a high-value agent than in treatments with a low-cost agent.

This paper is organized as follows. In the next section we outline the key theoretical concepts of network formation with heterogeneous agents and incomplete information (Sections 2.1 and 2.2). We present our experimental design, the experimental parameters and procedures (Sections 2.3 and 2.5), and discuss theoretical predictions (Section 2.4). Section 3 presents results on the empirical frequency of Nash networks (Section 3.1), stars (Section 3.2), the efficiency of observed networks (Section 3.3), their stability (Section 3.4), and the determinants of individual linking behavior (Section 3.5). Section 4 concludes. Appendix A contains proofs and Appendix B contains the instructions.

\section{Model, experimental design, and theoretical predictions}

We extend the two-way flow model with decay of Bala and Goyal (2000) to allow for agents that differ in costs or benefits of linking. For alternative approaches to heterogeneity in networks see the models of Johnson and Gilles (2000), and Haller and Sarangi (2005), in which links rather than agents vary in costs or reliability. A related model of Galeotti et al. (2006) studies network formation among heterogeneous agents without decay.

\subsection{Basic network concepts}

Let $N=\{1, \ldots, n\}$ denote the set of agents, with distinct generic members $i$ and $j$. Any agent can make a link to any other agent. Agent $i$ 's links can be represented by the linking vector $g_{i}=\left(g_{i 1}, \ldots, g_{i n}\right)$ such that $g_{i i}=0$ and $g_{i j} \in\{0,1\}$ for each $j \in N \backslash\{i\}$ where $g_{i j}=1$ if and only if $i$ made a link with $j$. The collection of all agents $N$ and all their links $\left\{(i, j) ; g_{i j}=1\right\}$ constitute a directed graph, or a network, which can be represented by the matrix $g=\left(g_{1}, \ldots, g_{n}\right)$.

Graphically we represent agents by small circles and their links by arrows. A link made by agent $i$ to agent $j$ is represented by a line starting at $i$ with the arrowhead pointing towards $j$. Fig. 1 shows an example of a network, where agents 4 and 6 made no links, agent 1 made a link to agents 2 and 4, and agents 2, 3 and 5 made a link to agent 4 .

Two agents are linked whenever at least one of them made (maintains) a link to the other. It is thus useful to define the closure of $g$ : this is a non-directed network $\bar{g}=\mathrm{cl}(g)$, defined by $\bar{g}_{i j}=\max \left\{g_{i j}, g_{j i}\right\}$ for each $i, j \in N$. A path of length $k$ between $i$ and $j$ is a sequence of distinct agents $\left(i, j_{1}, \ldots, j_{k-1}, j\right)$, such that $\bar{g}_{i j_{1}}=\bar{g}_{j_{1} j_{2}}=\cdots=\bar{g}_{j_{k-1} j}=1$. If at least one path exists between $i$ and $j$ then $j$ is accessible for $i$ (and vice versa), otherwise $j$ is inaccessible for $i$ (and vice versa). A path $\left(j_{1}, \ldots, j_{k}\right)$ is a cycle of length $k$ if $k \geqslant 3$ and $\bar{g}_{j_{k} j_{1}}=1$. The distance between $i$ and $j, i \neq j$, denoted $d(i, j$; $g$ ), is the length of the shortest path between $i$ and $j$. If $j$ is inaccessible for $i$ then the distance is $d(i, j ; g)=\infty$. If $i$ and $j$ are (directly) linked, that is, if $\bar{g}_{i j}=1$, then $d(i, j ; g)=1$. For completeness we set $d(i, i ; g)=0$. The degree of an agent $i$, deg ${ }_{i}(g)=\sum_{j \neq i} \bar{g}_{j i}$, is the number of other agents with whom she is linked. Fig. 1 shows a network with, among else, two paths between agents 1 and $5,(1,4,5)$ and $(1,2,4,5)$, and a cycle $(1,2,4,1)$. The distance between agents 1 and $5, d(1,5)=2$, is implied by the length of the shortest path between them, $(1,4,5)$. Agent 2 is linked with agents 1 and 4 , thus her degree is 2 .

A non-empty subset of agents $M \subset N$ is a component of network $g$ if every two distinct members of $M$ access each other but no agent in $M$ accesses any agent in $N \backslash M$. An agent having no links in the closure $\bar{g}$ is isolated, and forms a component consisting of one member. A network is connected if each agent accesses all other agents. In a connected network there are no isolated agents and the unique component consists of the whole set of agents $N$. A network is minimally sponsored if $g_{i j}=1$ implies $g_{j i}=0$ for any $i$ and $j$, that is, if every link is maintained by exactly one agent. A network is minimally connected if it is minimally sponsored, connected and contains no cycles. In such a network there is a unique path between every pair of distinct agents. For example, all networks shown in Figs. 4-5 below are minimally connected. The network in Fig. 1 is not minimally connected as it contains a cycle and an isolated agent.

\footnotetext{
8 See Fehr and Schmidt (1999) for an analysis of envy and guilt in general games, and Falk and Kosfeld (2005) for an application to networks.
} 
$6^{\circ}$

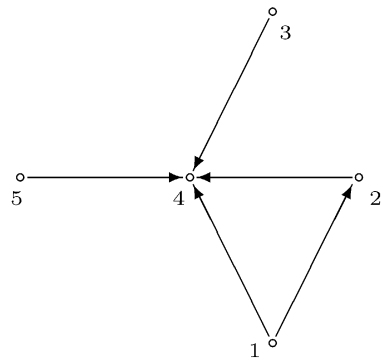

Fig. 1. Example of a network of directed links among 6 agents.

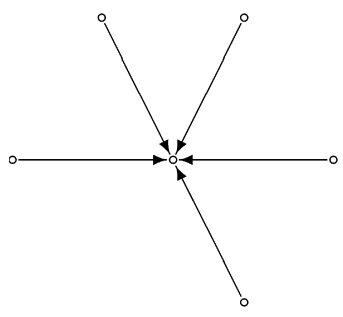

a) PSS b) EN

Fig. 2. Strict (Bayesian) Nash networks for treatments B and Vi.

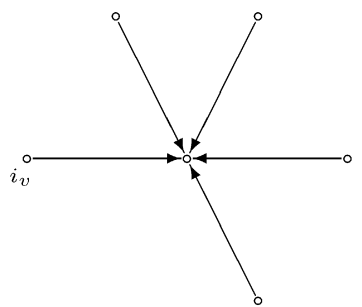

a) $\mathrm{PSS}_{0}$

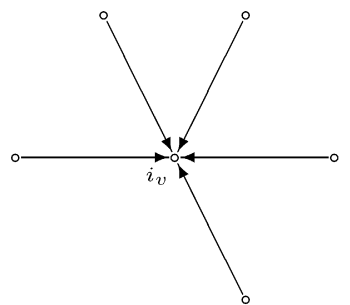

b) $\mathrm{PSS}_{v}$

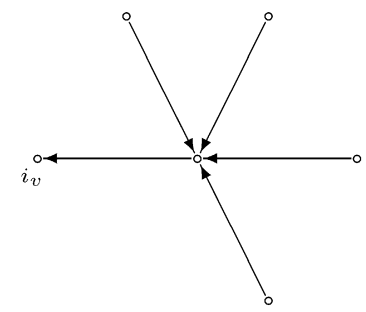

c) $\mathrm{PSS}_{o}^{-v}$

Fig. 3. Strict Nash networks for treatment V.

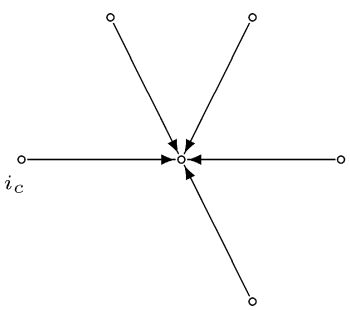

a) $\mathrm{PSS}_{0}$

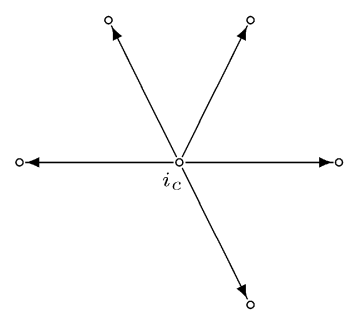

c) $\mathrm{CSS}_{C}$

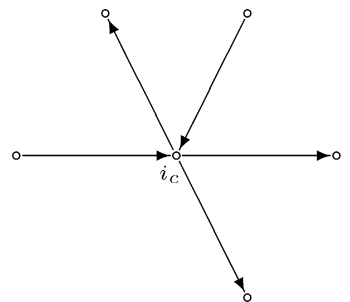

b) $\mathrm{MSS}_{c}\left(\right.$ incl. $\mathrm{PSS}_{c}$ )

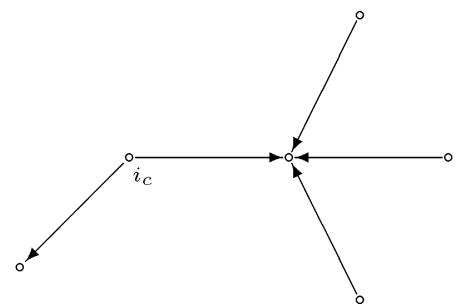

d) $\mathrm{LS}_{\mathrm{CO}}^{1}$

Fig. 4. Strict (Bayesian) Nash networks for treatments C, Ci and CVi. 


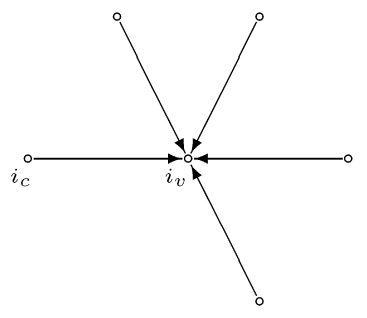

a) $\mathrm{PSS}_{v}$

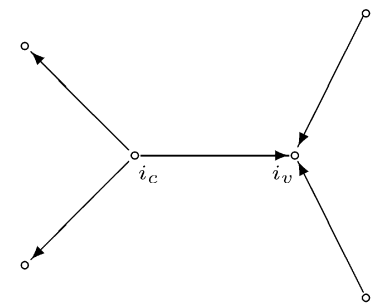

c) $\mathrm{LS}_{c v}^{1}$

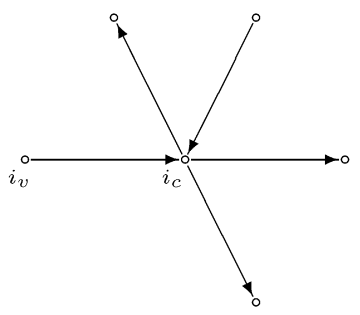

b) $\mathrm{MSS}_{\mathrm{C}}$

Fig. 5. Strict Nash networks for treatment CV.

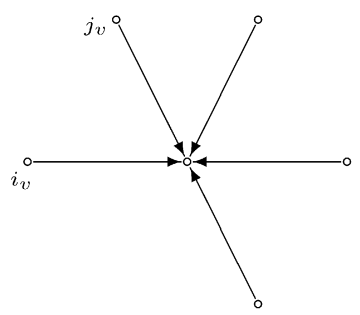

a) $\mathrm{PSS}_{0}$

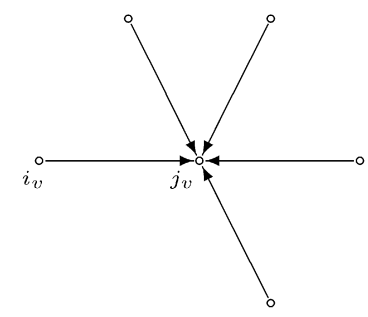

b) $\mathrm{PSS}_{v}$

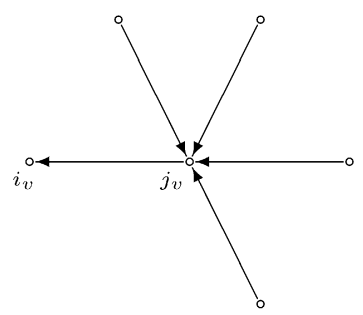

c) $\mathrm{PSS}_{v}^{-v}$

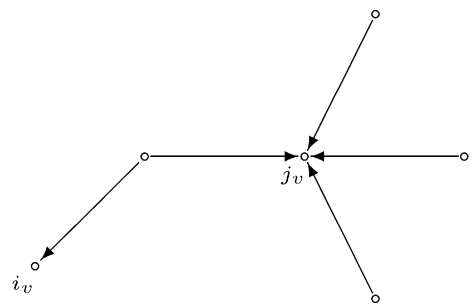

d) $\operatorname{LS}_{o v}^{v}$

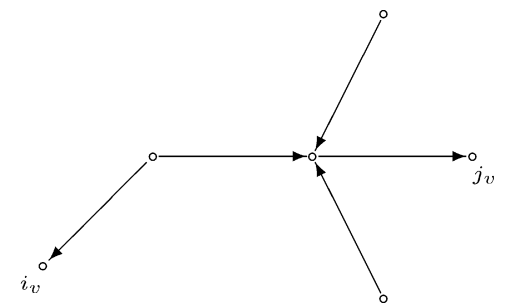

e) $\operatorname{LS}_{o o}^{v-v}$

Fig. 6. Strict Nash networks for treatment VV.

An agent that has exactly one link in the closure $\bar{g}$ is called periphery. We say that agent $i$ sponsors the link with agent $j$ when $g_{i j}=1$ and $g_{j i}=0$. For later reference it is convenient to identify some prominent classes of networks. For a graphical representation of some of the following networks, see Figs. 2-6.

Empty network(EN): No links are made, $g_{i j}=0$ for all $i$ and $j$.

Complete network $(C N)$ : All links are made, $\bar{g}_{i j}=1$ for all $i$ and $j$.

Wheel network $(W N)$ : The links of the network form a cycle of length $n$ spanning all agents.

$\operatorname{Star}(S)$ : There is one central agent $i$. Each other agent $j \neq i$ is periphery, linked only with the central agent $i$. The direction of links is arbitrary. Formally, $\bar{g}_{i k}=1$ and $\bar{g}_{j k}=0$ for $j, k \neq i$.

Minimally-sponsored star(MSS): A star where each link is sponsored either by the center agent $i$ or by the periphery agent. Formally, $g_{k i}=1 \Leftrightarrow g_{i k}=0$ and $g_{k j}=0$ for all $j, k \neq i$.

Periphery-sponsored star(PSS): A minimally-sponsored star where all links with the central agent $i$ are sponsored by the periphery agents. Formally, $g_{k i}=1, g_{i k}=0$ and $g_{k j}=0$ for all $j, k \neq i$.

Center-sponsored star(CSS): A minimally-sponsored star where the central agent $i$ sponsors all the links. Formally, $g_{k i}=0$, $g_{i k}=1$ and $g_{k j}=0$ for all $j, k \neq i$.

Linked star(LS): There are two linked non-periphery agents $i$ and $j$ while all other agents are periphery, linked with either $i$ or $j$. Formally, $\bar{g}_{i j}=1, \bar{g}_{k i}=1$ or $\bar{g}_{k j}=1$ but not both, and $\bar{g}_{k l}=0$ for $k, l \notin\{i, j\}$. 
Table 1

Experimental parameters.

a) Linking costs and values of different agent types

\begin{tabular}{lcc}
\hline & Cost per link made & Value to other agents \\
\hline Normal agent & 24 & 16 \\
Low cost agent & 7 & 16 \\
High value agent & 24 & 32 \\
\hline
\end{tabular}

b) Benefits (per agent accessed) from accessing different types of agents at different distances

\begin{tabular}{llllrrr}
\hline Distance & 1 & 2 & 3 & 4 & 5 & 0 \\
\hline Normal or low cost agent & 16 & 12 & 9 & 7 & 5 \\
High value agent & 32 & 24 & 18 & 14 & 0 \\
\hline
\end{tabular}

\subsection{Heterogeneous agents, two-way flow of benefits, and decay}

Suppose each agent $i$ provides some service (the quality of which depends on personal skill or information) that has positive value $v_{i}$ to others. Agents can materialize these values by (simultaneously) forming links. Each agent $i$ incurs positive costs $c_{i}$ for every link she forms (capturing the time, effort, or money invested to form and maintain the link). When there is a link between agents $i$ and $j$, made either by $i$ or $j$ (or by both), both enjoy the other's value. Establishing a link therefore does not require mutual consent and the benefits flow both ways. Agents benefit also from any other agent they access through the network although the benefit decreases with distance (reflecting the idea that information may become less accurate, or less valuable as it propagates through the network).

Formally, the benefit that agent $i$ extracts from agent $j$ depends on the value $v_{j}$ as well as on the distance $d(i, j$; $g$ ) as given by the decay function $\Phi\left(v_{j}, d(i, j ; g)\right)$. We assume that $\Phi: \mathbb{R}_{+} \times\{\{0,1,2, \ldots, n-1\} \cup\{\infty\}\} \rightarrow \mathbb{R}$ is strictly increasing in value and decreasing in distance, with $\Phi\left(v_{j}, 0\right)=\Phi\left(v_{j}, \infty\right)=0$, that is, an agent receives no benefit from herself nor from those other agents she cannot access.

As in Bala and Goyal (2000) we assume a linear payoff function. The payoff to agent $i$ is the sum of the benefits accessed through the network minus the costs of links she maintains. Let $\mu_{i}(g)=\left|\left\{j \in N ; g_{i j}=1\right\}\right|$ be the number of links that agent $i$ maintains. The payoff to agent $i$ in the network $g$ is given by

$$
\pi_{i}(g)=\sum_{j \in N} \Phi\left(v_{j}, d(i, j ; g)\right)-\mu_{i}(g) c_{i}
$$

In the following we assume an exponential decay function $\Phi(v, d)=\delta^{d} v(\delta \in(0,1))$ as in Jackson and Wolinsky (1996) and Bala and Goyal (2000).

\subsection{Experimental parameters and design}

To separate the effects of heterogeneity in linking costs and heterogeneity in values we consider five situations. In the homogeneous case, all (normal) agents have identical costs and values. In the low-cost case, one (low-cost) agent has lower linking costs while all other (normal) agents are as in the homogeneous case. In the high-value case, the value of one (high-value) agent exceeds that of other (normal) agents who are as in the homogeneous case. We also study the situation with one low-cost and one high-value agent while keeping all other parameters identical to the homogeneous case. Most theoretical models in network formation assume complete information. In reality, however, this assumption is often not satisfied. Therefore, in addition to the complete information case, we analyze network formation with incomplete information about types (normal, low-cost, high-value). In the incomplete information case each agent only knows her own type and the distribution types. We always assume complete information about the network. ${ }^{9}$ To check the robustness of our results, we also investigate the case with two high-value agents instead of only one. ${ }^{10}$ In all cases we study groups of $n=6$ agents.

In summary, we investigate network formation behavior in five cases that differ with respect to heterogeneity implementing eight treatments (where 'i' stands for incomplete information):

(B) baseline treatment with groups of 6 normal agents,

(C \& Ci) treatments with groups of 5 normal agents and 1 low-cost agent,

(V \& Vi) treatments with groups of 5 normal agents and 1 high-value agent,

(VV) treatment with groups of 4 normal agents and 2 high-value agents, and

(CV \& CVi) treatments with groups of 4 normal agents, 1 low-cost agent, and 1 high-value agent.

The parameter values concerning linking costs, agents' values, and decay used in our experiments are depicted in Table 1.

\footnotetext{
9 There are many interesting kinds of incomplete information conceivable in network formation games. For instance, agents may also only have incomplete information about the actual network structure. We leave the study of these cases for future research.

10 We are thankful to an anonymous referee for suggesting this case.
} 
The choice of parameter values is guided by our interest in studying the (non-)formation of star networks. The assumed decay of benefits is a discrete approximation of $\delta^{d} v$ with $\delta=3 / 4$, given the values. In the homogeneous case (B) the chosen parameter values are theoretically most favorable for the formation of stars. With $c=24$ and $v=16$ all periphery-sponsored stars are the only Nash networks, together with the empty network (see Propositions 5.3 and 5.4 in Bala and Goyal, 2000). For the low-cost case (C \& Ci) the implemented low linking cost, $c^{l}=7$, implies that all minimally-sponsored stars with agent $i_{c}$ in the center as well as periphery sponsored stars with any agent in the center are Nash networks, whereas the empty network is not. Intuitively, this makes it more likely (relative to the homogeneous case) that a star network is established and that agent $i_{c}$ is in its center. For the high-value case $(\mathrm{V} \& \mathrm{Vi})$ the selected value $v^{h}=32$ guarantees that all periphery-sponsored stars, with any agent in the center, are Nash networks, but the empty network is not. Intuitively, this makes it again more likely (relative to the homogeneous case) that a star network is established where all agents are equally likely to be in its center. In the next section we formally present the theoretical results of the discussed cases as well as the two more complex cases with one low-cost agent $i_{c}$, one high-value agent $i_{v}$, and $n-2$ normal agents (CV \& $\mathrm{CVi}$ ) and two high-value agents $i_{v}$ and $j_{v}$ and $n-2$ normal agents (VV).

The network game is repeated for 30 rounds within each group. In each round, subjects first simultaneously make their linking decisions and then each subject is informed about the established network, her total benefits, her costs, and her payoff. Groups are fixed throughout the experiment and in each group each subject keeps a unique identification tag. In all treatments the player types are fixed throughout the experiment, that is, each subject stays with the initially allocated role for all 30 rounds. In treatments $\mathrm{B}, \mathrm{V}, \mathrm{VV}, \mathrm{C}$, and $\mathrm{CV}$, the type of each agent is publicly announced at the beginning of the first round of the experiment. In treatments $\mathrm{Ci}, \mathrm{Vi}$, and $\mathrm{CVi}$, the collection of types in the group is publicly announced at the beginning of the first round of the experiment, but the type of each agent remains private information throughout the experiment. Treatments $\mathrm{Ci}, \mathrm{Vi}$, and $\mathrm{CVi}$ differ from treatments $\mathrm{C}, \mathrm{V}$, and $\mathrm{CV}$, respectively, only in terms of the quality of information.

\subsection{Equilibrium and efficiency analyses}

Below we provide a complete characterization of the set of (Bayesian) Nash networks assuming selfish preferences. In addition, we show that most equilibrium networks are robust to the introduction of social preferences, with some important exceptions. ${ }^{11}$

Following previous literature on network formation games we consider only pure strategies (Jackson, 2004). The set of pure strategies of agent $i, \mathcal{G}_{i}$, is the set of all her possible linking vectors $g_{i}$. The strategy space of all agents is given by $\mathcal{G}=\mathcal{G}_{1} \times \cdots \times \mathcal{G}_{n}$. Any profile of strategies $g=\left(g_{1}, \ldots, g_{n}\right)$ constitutes a (directed) network. Agents' benefits depend on $\bar{g}$, the undirected closure of $g$.

If agents' values and linking costs and the decay function are common knowledge then the network formation game is played under complete information. In this case we define Nash networks to be the networks that are established in the (pure-strategy) Nash equilibria of the game $\left\langle N, \mathcal{G},\left(\pi_{i}\right)_{i=1}^{n}\right\rangle$. Given a network $g$ let $g_{-i}$ denote the network obtained when all links maintained by agent $i$ are removed. The network $g$ can thus be written as $g=g_{i} \oplus g_{-i}$, where the symbol ' $\oplus$ ' indicates that $g$ is formed by the union of links in $g_{i}$ and $g_{-i}$. A strategy $g_{i}$ is a best response of agent $i$ to network $g$ if

$$
\pi_{i}\left(g_{i} \oplus g_{-i}\right) \geqslant \pi_{i}\left(g_{i}^{\prime} \oplus g_{-i}\right) \text { for all } g_{i}^{\prime} \in \mathcal{G}_{i} .
$$

The set of all best responses of agent $i$ to $g$ is denoted by $B R_{i}\left(g_{-i}\right)$. A network $g=\left(g_{1}, \ldots, g_{n}\right)$ is a Nash network if $g_{i} \in B R_{i}\left(g_{-i}\right)$ for each $i$. A Nash network is strict Nash if $\left|B R_{i}\left(g_{-i}\right)\right|=1$ for each $i$, i.e. each agent is playing her unique best response to the network established by the other agents.

The definition of equilibrium networks is more intricate for network formation games with incomplete information. ${ }^{12}$ For convenience, the definition we give here is limited to the setup employed in our experiments. Let the decay function $\Phi$ be common knowledge. Let the profile of agents' value-cost pairs $\theta=\left(\left(v_{1}, c_{1}\right), \ldots,\left(v_{n}, c_{n}\right)\right)$ be randomly drawn from some finite space of profiles $\Theta$ and let the ex-ante probability distribution $p$ over $\Theta$ be common knowledge. We denote by $v_{i}(\theta)$ and $c_{i}(\theta)$ the value and linking cost of agent $i$ given a profile $\theta$. For any profile $\theta \in \Theta$ and any network $g$ the payoff to agent $i$ is given by

$$
u_{i}(g ; \theta)=\sum_{j \in N} \Phi\left(v_{j}(\theta), d(i, j ; g)\right)-\mu_{i}(g) c_{i}(\theta)
$$

Once a profile $\theta \in \Theta$ is drawn each agent $i$ learns her own value and linking cost, $\theta_{i}=\left(v_{i}(\theta), c_{i}(\theta)\right)$, which permits her to calculate her (subjective) beliefs $p_{i}^{\theta_{i}}$ over $\Theta$ and her expected payoff $E_{i} u_{i}\left(g ; \theta_{i}\right)=\sum_{\theta^{\prime} \in \Theta} u_{i}\left(g ; \theta^{\prime}\right) p_{i}^{\theta_{i}}\left[\theta^{\prime}\right]$ in the network $g$. Formally, this defines the Bayesian game $\left\langle N, \mathcal{G}, \Theta,\left(\left(p_{i}^{\theta_{i}}\right)_{\theta \in \Theta}\right)_{i=1}^{n},\left(u_{i}\right)_{i=1}^{n}\right\rangle$.

\footnotetext{
11 Clearly the set of (Bayesian) Nash networks depends on the parameters of the model. However, a full characterization of equilibria for arbitrary parameter values is beyond the scope of this paper. For attempts of a general characterization of equilibrium networks with heterogeneous agents, see Bala and Goyal (2000) and Galeotti et al. (2006). However, also in those papers the set of Nash networks is characterized completely only for certain ranges of parameters (see also Haller et al., 2007).

12 To the best of our knowledge, network formation under incomplete information has been studied only by McBride (2008).
} 
The set of equilibrium networks may depend on the realized value-cost profile. A network $g$ is a Bayesian Nash network given profile $\theta \in \Theta$ if, for each agent $i$,

$$
E_{i} u_{i}\left(g_{i} \oplus g_{-i} ; \theta_{i}\right) \geqslant E_{i} u_{i}\left(g_{i}^{\prime} \oplus g_{-i} ; \theta_{i}\right) \text { for all } g_{i}^{\prime} \in \mathcal{G}_{i} \backslash\left\{g_{i}\right\} .
$$

In a Bayesian Nash network no agent can increase her expected payoff given her beliefs about the profile of values and linking costs among the agents. ${ }^{13}$ Network $g$ is strict Bayesian Nash, given $\theta$, if all inequalities (3) are strict.

Below we use the subscripts $c, v$, and $o$ to indicate a low-cost agent, a high-value, or a (normal) other agent, respectively For $x \in\{c, v, o\}$ in the center, a minimally-sponsored star is denoted MSS $_{x}$, a periphery-sponsored star is denoted PSS ${ }_{x}$, and a center-sponsored star is denoted $\mathrm{CSS}_{x}$. PSS ${ }_{x}^{-v}$ denotes a star with an agent of type $x$ in the center that is peripherysponsored except for the center sponsoring links to one or two high-value agents (see Figs. $3 \mathrm{c}$ and $6 \mathrm{c}$ ). $L S_{x y}^{p}$ denotes a linked star with an agent of type $x$ in one center sponsoring links with $p$ periphery agents, and an agent of type $y$ in the other periphery-sponsored center linked with $4-p$ periphery agents $\left(\mathrm{LS}_{c o}^{1}, \mathrm{LS}_{c v}^{2}\right.$ and $\mathrm{LS}_{c v}^{1}$ are shown in Figs. $4 \mathrm{~d}, 5 \mathrm{c}$, and $5 \mathrm{~d}$, respectively). $\mathrm{LS}_{o v}^{v}$ denotes a linked star $\mathrm{LS}_{o v}^{1}$ in which the first center sponsors a link with the periphery high-value agent, and $\mathrm{LS}_{o o}^{v-v}$ denotes a linked star $\mathrm{LS}_{o o}^{1}$ in which each center sponsors a link with one periphery high-value agent (see Fig. 6d, e). In all the linked stars mentioned below, the link between the two central agents is sponsored by the first, except for $\mathrm{LS}_{c v}^{2}$ where this link can be sponsored by any central agent.

Propositions 1 and 2 provide complete characterizations of the strict (Bayesian) Nash networks for the one-shot network formation games in each of our treatments. Proofs can be found in Appendix A, and illustrations of the equilibrium networks are given in Figs. 2-6.

Proposition 1. The following are the only strict Nash networks for the complete information treatments:

$B:$ all PSS and the EN.

$V:$ all PSS and all $P S S_{0}^{-v}$.

C: all PSS, all MSS ${ }_{c}$ including the $\operatorname{CSS}_{c}$, and all $L S_{c o}^{1}$.

$C V$ : the $P S S_{v}$, all MSS $S_{c}$ including the $\operatorname{CSS}_{c}$, and all $L S_{c v}^{1}$ and $L S_{c v}^{2}$.

$V V$ : all PSS, all PSS $S_{o}^{-v}$, both PSS ${ }_{v}^{-v}$, and all $L S_{o v}^{v}$ and $L S_{o o}^{v-v}$.

Proposition 2. The following are the only strict Bayesian Nash networks for the incomplete information treatments, given any feasible allocation of types:

Vi: all PSS and the EN.

Ci, CVi: all PSS, all MSS $S_{c}$ including the $C S S_{c}$, and all $L S_{c o}^{1}$ and $L S_{c v}^{1}$.

Predictions regarding the impact of information on network formation are obtained by comparing the set of equilibrium networks across the treatments with identical type distributions. Limiting information changes the set of equilibrium networks in the treatments with a high-value agent, but not in the treatment with only one low-cost agent. This suggests, in particular, that behavior in $\mathrm{C}$ should be similar to that in $\mathrm{Ci}$. With repetition, however, agents may overcome the uncertainty about types in any incomplete information treatment. An interesting question, therefore, is whether in the experiment behavior in later rounds of an incomplete information treatment will be similar to behavior in the corresponding complete information treatment.

Efficiency can be measured with the sum of agents' payoffs. Let $w: \mathcal{G} \rightarrow \mathbb{R}$ be defined as $w(g)=\sum_{i=1}^{n} \pi_{i}(g)$. A network $g$ is efficient if $w(g) \geqslant w\left(g^{\prime}\right)$ for all $g^{\prime} \in \mathcal{G}$. For the linear payoffs in Eq. (1) the efficient network is the one which maximizes the total benefits of all agents, less the aggregate cost of links of the network. Proposition 3 shows that star networks have the best ratio between the number of links and the aggregate distance between agents.

Proposition 3. The following are the only efficient networks for the different treatments:

B: all MSS.

$V, V i, V V:$ all MSS

C, Ci, CV, CVi: the $\operatorname{CSS}_{C}$.

The proof of Proposition 3 is provided in Appendix A. In the baseline treatment B, each periphery-sponsored star network is Nash and efficient. In treatment VV, all PSS ${ }_{v}$ and $\mathrm{PSS}_{v}^{-v}$ are Nash and efficient. In all other treatments there is a unique efficient (Bayesian) Nash network. To summarize, in every treatment the efficient equilibrium network is a star network. For

\footnotetext{
13 The relation between ex-ante defined Bayesian equilibria and the "interim" defined Bayesian Nash networks is as follows. A (pure) strategy profile $s: \Theta \rightarrow \mathcal{G}$ is a Bayesian equilibrium of our network formation game if and only if $s(\theta)$ is a Bayesian Nash network given any allocation $\theta \in \Theta$. See, e.g., Fudenberg and Tirole (1991) for discussion on the equivalence between ex-ante and "interim" formulations of equilibria in Bayesian games.
} 
later use, w also define the relative efficiency of a network $g \in \mathcal{G}$ to be the ratio $w(g) / w\left(g^{*}\right)$, where $g^{*} \in \mathcal{G}$ is the efficient network. The two benchmark relative efficiencies are 0 for the empty network and 1 for the efficient network.

Finally, payoff inequalities may destabilize a network if it is established by agents that are inequality averse. In order to study the impact of inequality aversion for network formation we use the model of Fehr and Schmidt (1999). In this model, each player $i$ is characterized by two coefficients of inequality aversion $\alpha_{i}$ and $\beta_{i}$, satisfying

$$
0 \leqslant \beta_{i}<1 \text { and } \beta_{i} \leqslant \alpha_{i},
$$

where $\alpha_{i}$ describes player $i$ 's aversion to disadvantageous inequality (envy) and $\beta_{i}$ describes her aversion to advantageous inequality (guilt). For each vector of (monetary) earnings $\pi=\left(\pi_{1}, \ldots, \pi_{n}\right)$ the utility of player $i$ is given by

$$
u_{i}(\pi)=\pi_{i}-\frac{\alpha_{i}}{n-1} \sum_{j} \max \left\{\pi_{j}-\pi_{i}, 0\right\}-\frac{\beta_{i}}{n-1} \sum_{j} \max \left\{\pi_{i}-\pi_{j}, 0\right\} .
$$

Note that when the inequality among players increases, the utility of a player with positive coefficients of inequality aversion decreases. Fehr and Schmidt (1999) use several experimental data sets to come up with the following "natural" bounds on coefficients of inequality aversion:

$$
0 \leqslant \alpha_{i} \leqslant 4 \text { and } 0 \leqslant \beta_{i} \leqslant 0.6
$$

Proposition 4 below shows, for our complete information treatments, that most networks which are strict Nash when formed by agents with standard preferences remain strict Nash when formed by agents with natural levels of inequality aversion. ${ }^{14}$

Proposition 4. The strict Nash networks characterized by Proposition 1 remain strict Nash networks for natural levels of inequality aversion, except:

C: CSS $_{c}$ is no longer SNN if $\alpha_{c}>1.5+1.2 \beta$ and MSS $_{c}$ where the central agent sponsors four links are no longer SNN if $\alpha_{c}>2.2+2.2 \beta$. $C V:$ CSS $_{c}$ is no longer SNN if $\alpha_{c}>1.7+2 \beta$, MSS $_{c}$ where the central agent sponsors four links are no longer SNN if $\alpha_{c}>3.3+4.7 \beta$, and $L S_{c v}^{2}$ where $i_{v}$ sponsors the link with $i_{c}$ are no longer $S N N$ if $\alpha_{v}>1.1+0.7 \beta$.

$V V: P S S_{o}^{-v}$ where the central agent sponsors two links are no longer SNN if $\alpha_{o}>1.7+4.3 \beta$, and $L S_{o v}^{v}$ and $L S_{o o}^{v-v}$ are no longer SNN if $\alpha_{0}>0.7+0.8 \beta$ and, respectively, $\alpha_{o}>0.7+0.7 \beta$ for central agents 0 .

The intuition for these results is as follows. Recall that an inequality averse agent is willing to deviate from an equilibrium and sacrifice part of her earnings only if that substantially decreases the total inequality she experiences. For most of the Nash networks listed in Proposition 1 a unilateral deviation by one of the agents results in a loss of earnings and does not sufficiently decrease, or may even increase, the inequality she experiences (e.g., when a peripheral agent unilaterally deviates by removing her link to the high-value agent in the center). An exception is the removal of links by the low-cost agent in the center of the $\mathrm{CSS}_{c}$ and some $\mathrm{MSS}_{c}$ networks. In such networks, the center earns much less than the peripheral agents she supports. By removing links, the center lowers others' earnings substantially more than her own, thus lowering her material payoffs but at the same time reducing feelings of envy.

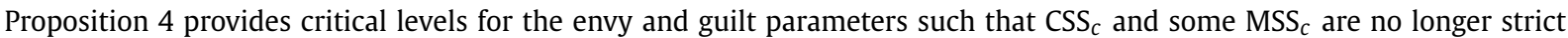
Nash. As we report below, the envy and guilt estimates for our data set are close to these critical levels, which explains one of our main treatment effects: the lack of star formation in the low-cost treatments compared to the abundance of stars in the high-value treatments.

\subsection{Experimental procedures}

The experimental sessions were conducted in Spring of 2003 and 2007 at the CREED laboratory at the University of Amsterdam and at the Social Science Experimental Laboratory at the California Institute of Technology. In total 264 subjects participated. Each experimental session lasted between 45 and 90 minutes. Subjects' total earnings were determined by the sum of the points earned over all the rounds, using a conversion rate of 70 points per Euro or US dollar (the benefits and costs listed in Table 1 are all in points). The average earnings were 22.8 Euros or (roughly) USD 25 at the time the experiments were conducted. Subjects were recruited through notices on bulletin boards and through email announcements. Each subject participated in only one session and none had previously participated in a similar experiment. To ensure anonymity, at least twelve subjects were recruited for every session and were randomly divided into at least two independent groups. They were seated in separated cubicles, which ensured anonymity during the experiment. We consider each group as one

\footnotetext{
14 The partial characterization of the equilibrium networks is sufficient for our analysis. It is, however, interesting to note that particular combinations of inequality aversion parameters lead to new equilibrium networks even when all agents are equally inequity averse. To illustrate, the empty network is strict Nash in treatments C, V and VV when $\alpha_{i}=4$ and $\beta_{i}=0.6$ and various linked stars are strict Nash in all complete information treatments when $\alpha_{i}=\beta_{i}=0.6$. Curiously, wheel networks are strict Nash in treatment B whenever $\beta_{i} \geqslant 0.8$ and a disconnected network with only one link, sponsored by the low-cost agent, is strict Nash in treatment $\mathrm{C}$ when $\alpha_{i}=4$ and $\beta_{i}=0$.
} 
independent observation. The number of groups in treatments $\mathrm{B}, \mathrm{C}, \mathrm{Ci}, \mathrm{V}, \mathrm{Vi}, \mathrm{VV}, \mathrm{CV}$, and $\mathrm{CVi}$ are 7, 6, 6, 4, 6, 5, 4, and 6, respectively.

At the beginning of a session, subjects were told the rules of conduct and provided with detailed instructions. The instructions were read aloud and shown on the computer screen. ${ }^{15}$ After having finished the instructions, subjects received a printed summary and were asked to complete a questionnaire designed to test their understanding of the network formation game, the payoff calculations, and the computer interface. Once all subjects had correctly answered the questionnaire, we conducted a single practice round (without providing feedback about others' choices). The experiment started after all subjects confirmed they had no further questions.

Care was taken to minimize differences in the instructions for different treatments. ${ }^{16}$ In all treatments, subjects knew the size of their group, the numbers of normal, low-cost, and high-value types in their group, the type-conditional payoff functions, the information condition, and the number of rounds. To increase anonymity and avoid suggestive framing, each subject's screen displayed herself as "Me" and the other five members in her group as "A," "B," "C," "D," and "E." Each letter corresponded to the same member in the group throughout the session, and this was common knowledge. The game was neutrally framed. Subjects types were named after colors: "green" (normal), "purple" (low-cost), or "blue" (high-value). The term "network" was also avoided, instead subjects were forming "links" with other subjects. In the complete information treatments, each subject observed the color of all other subjects in the group. In the incomplete information treatments, each subject only observed her own color. Each subject's color remained the same during all 30 periods of the experiment.

\section{Results}

We first present results concerning the occurrence of equilibrium networks in the different treatments. Then we investigate whether star networks are formed and how the frequency of stars (if any) varies across treatments and over time. We briefly discuss the efficiency and stability of the observed networks. Finally, we study how individual behavior accounts for the observed networks. For convenience we use the classification of star architectures introduced in Sections 2.1 and 2.4. If not otherwise stated all statistical tests are based on independent observations, i.e. groups.

We begin with a few preliminary observations. Subjects in our experiments actively link with each other: across all treatments and all rounds we never observe the empty network. Neither do we observe the complete network, which indicates that there is no excessive 'over-linking.' Furthermore, we observe that between 11 and $14 \%$ of networks in treatments B, $\mathrm{C}$ and $\mathrm{Ci}$ are minimally connected whereas in treatments $\mathrm{V}, \mathrm{Vi}, \mathrm{VV}, \mathrm{CV}$ and $\mathrm{CVi}$ their frequencies are between 43 and $60 \%$. Since non-empty equilibrium networks are minimally connected (see Appendix A) these frequencies suggest that the presence of a high-value agent facilitates formation of equilibrium networks.

\subsection{Do subjects form equilibrium networks?}

Table 2 depicts the frequency of (Bayesian) Nash networks in all treatments across all rounds. The differences across treatments are quite striking. In the baseline treatment $B$ with only homogeneous agents not a single Nash network is formed. A similar conclusion holds for treatments with one low-cost agent. In C and $\mathrm{Ci}$, respectively, only 2.2 and $8.9 \%$ of all observed networks are (Bayesian) Nash. However, introducing at least one high-value agent has a dramatic effect on the frequency of equilibrium networks. In $\mathrm{V}, \mathrm{VV}$, and $\mathrm{Vi}, 40.8,50.7$, and $51.1 \%$ of all observed networks are (Bayesian) Nash and for CV and CVi the ratios are 33.3 and $26.7 \%$, respectively.

Considering data from only the last five rounds indicates even larger differences across treatments. The relative frequency of (Bayesian) Nash networks in V, VV, Vi, CV, and CVi, respectively, is 75.0, 92, 83.3, 95.0, and 66.7\%. In stark contrast, in C and $\mathrm{Ci}$ only 10.0 and $16.7 \%$ of all networks are (Bayesian) Nash. In B no Nash networks are formed at all. In the treatments where a high-value agent is present subjects tend to form an equilibrium network whereas this is not the case in the other treatments. ${ }^{17}$

\subsection{Do subjects form star networks?}

Fig. 7 depicts the frequency of the different star architectures for all seven treatments aggregated across the first five rounds (panel a) and the last five rounds (panel b). Very few star structures are formed in early rounds. In treatments $\mathrm{B}, \mathrm{Ci}$, CV and CVi, no stars are observed at all. In treatment C 10\% (3/30) of all networks are stars, all of them have the low cost

\footnotetext{
15 The experiment and the questionnaire were computerized using software developed at CREED by Jos Theelen.

16 Instructions can be found in Appendix B with the parts that differ between treatments emphasized.

17 We calculated the Spearman rank order correlations between the average number of (Bayesian) Nash networks across groups and the round number. For treatments V, VV, Vi, CV, and CVi this correlation coefficient is larger than 0.86 and significant at $p<0.0001$ (two-sided tests). This clearly indicates that in these treatments more equilibrium networks are formed as agents gain experience. Interestingly, there is weak evidence of learning in treatment Ci, where the percentage of Bayesian Nash networks increases from $0.0 \%$ in the first 16 rounds to $16.7 \%$ in the last few rounds. This increase is statistically significant ( $\rho=0.84, p<0.0001$; two-sided test). Note, however, that in quantitative terms the number of Bayesian Nash networks in Ci is considerably less than in treatments with a high-value agent. No convergence towards Nash networks can be found in treatments B (because there are no Nash networks at all) and $C$ where the Spearman correlation coefficient is not significantly different from zero ( $p>0.2935$; two-sided tests).
} 
Table 2

Frequency of (Bayesian) Nash networks in the different treatments.

\begin{tabular}{|c|c|c|c|c|c|c|c|c|c|c|c|}
\hline \multirow[t]{2}{*}{ Treatment } & \multicolumn{9}{|c|}{ Equilibrium networks (in percent) } & \multirow[t]{2}{*}{ Total } & \multirow[t]{2}{*}{ \# Obs. } \\
\hline & EN & $\mathrm{PSS}_{o}$ & $\mathrm{PSS}_{v}$ & $\mathrm{PSS}_{0}^{-v}$ & $\mathrm{PSS}_{v}^{-v}$ & $\mathrm{MSS}_{c}$ & $\mathrm{LS}_{o v}$ & $\mathrm{LS}_{C o}$ & $\mathrm{LS}_{c v}$ & & \\
\hline B & 0.0 & 0.0 & & & & & & & & 0.0 & 210 \\
\hline C & & 0.0 & & & & 2.2 & & 0.0 & & 2.2 & 180 \\
\hline $\mathrm{Ci}$ & & 0.0 & & & & 8.9 & & 0.0 & & 8.9 & 180 \\
\hline V & & 0.0 & 40.8 & 0.0 & & & & & & 40.8 & 120 \\
\hline VV & & 0.0 & 30.0 & & 4.0 & & 16.7 & & & 50.7 & 150 \\
\hline Vi & 0.0 & 0.0 & 51.1 & & & & & & & 51.1 & 180 \\
\hline $\mathrm{CV}$ & & & 33.3 & & & 0.0 & & & & 33.3 & 120 \\
\hline $\mathrm{CVi}$ & & 0.0 & 17.8 & & & 5.0 & & & 3.9 & 26.7 & 180 \\
\hline
\end{tabular}

Note: $\mathrm{PSS}_{0}\left(\mathrm{PSS}_{v}\right)$ : periphery-sponsored star with a normal (high-value) type in the center; $\mathrm{PSS}_{0}^{-v}$ (PSS ${ }_{v}^{-v}$ ): star with a normal (one high-value) agent in the center that is periphery-sponsored except for the center sponsoring one link to the (other) high-value agent; MSS ${ }_{c}$ : minimally-sponsored star with the low-cost agent in the center; $\mathrm{LS}_{o v}\left(\mathrm{LS}_{c o}\right)\left[\mathrm{LS}_{c v}\right]$ : linked star with a high-value ([low-cost]) agent in one center and a normal [high-value] agent in the other center; EN: empty network. Empty cells indicate the network is not an equilibrium network in the corresponding treatment.

agent in the center with mixed sponsoring. Some stars are also formed in the treatments with at least one high-value agent (25\% (5/20) in V, 10\% (3/30) in Vi, and 4\% (1/25) in VV). Interestingly, all observed star networks in V and Vi are efficient equilibrium networks, i.e. periphery-sponsored stars with the high-value agent in the center.

Fig. 7b highlights the differences across treatments in the last five rounds. Comparing the results to those for the first five rounds indicates that repetition and experience can have a dramatic effect on formation of stars. In the baseline treatment with homogeneous agents only 2 out of 35 networks in the last five rounds are stars (5.7\%). ${ }^{18}$ A low frequency of stars is observed also in the treatments with one low cost agent, $\mathrm{C}$ and $\mathrm{Ci}$. In C only 5 networks out of 30 are stars (16.7\%); in all of them the low-cost agent is in the center. Interestingly, there are slightly more stars in the incomplete information case Ci, but their frequency is with $26.7 \%(8 / 30)$ still low, ${ }^{19}$ and the differences between the two information conditions are not significant ( $p=0.53$ using a $\chi^{2}$ test).

The results for the treatments with at least one high-value agent are very different. In all five treatments $\mathrm{V}, \mathrm{VV}, \mathrm{Vi}, \mathrm{CV}$, and CVi, stars are the prevailing network architecture. In V 75\% (15/20), in VV 56\% (14/25) and in Vi 83.3\% (25/30) of all networks are stars. The difference between the two information conditions $\mathrm{V}$ and $\mathrm{Vi}$ is not significant ( $p=0.71$ using a $\chi^{2}$ test). In CVi the frequency of stars is 60\% (18/30) while in CV their frequency is almost $100 \%$ (19/20). In this case, the difference between the information conditions is significant ( $p=0.01$ using a $\chi^{2}$ test). In other words, the introduction of private information about players' types significantly lowers the number of stars formed when both high-value and low-cost agents are present.

Remarkably, in V, VV, Vi, and CV all formed stars have the high-value agent in the center and most are periphery sponsored. In CVi such networks are formed in the majority of cases. ${ }^{20}$ It is worth noting that in VV and CVi also linked stars, which are inefficient equilibrium networks, are formed (VV: 36\% (9/25), CVi: 6.7\% (2/30)) in the last 5 rounds. From these results it is obvious that the presence of a high-value agent facilitates the formation of (linked) stars, but not necessarily efficient ones. ${ }^{21}$ Furthermore, as for the case of equilibrium networks, the dynamics of star formation are quite different across treatments. The Spearman rank order correlation of the average number of stars (of any form) across groups with the round is insignificant for treatments $\mathrm{B}$ and $\mathrm{C}(p \geqslant 0.2255$; two-sided tests). In all other treatments we observe significantly positive correlations $(\rho>0.80, p<0.001 \text {; two-sided tests })^{22}$

To summarize, stars (whether equilibrium or not) very rarely form in early rounds when subjects have little or no experience (independent of the treatment). This indicates that forming a star with a high-value or a low-cost agent in the center is not an obvious or focal outcome. With repetition, only a few stars are formed when agents are homogeneous or differ only in linking costs. In contrast, in treatments with at least one high-value agent, experienced agents often form stars, most frequently with the high-value agent in the center.

Given that stars only form in some of the treatments, asking for 'exact' stars may be too restrictive given the huge coordination problem subjects face in the experiment. We therefore also investigate the less restrictive measure of 'centrality' of a network.

\footnotetext{
18 Interestingly, also other prominent structures do not show up in this treatment. The complete network is never formed and the wheel is formed in only 2 out of 35 cases.

19 Note that none of the observed stars in C coincides with the efficient center-sponsored star where the low-cost agent sponsors all links. In Ci the majority (62.5\%; 5/8) of star networks are efficient $\mathrm{CSS}_{c}$ networks.

20 The efficient network in treatments $\mathrm{CV}$ and $\mathrm{CVi}$ is $\mathrm{CSS}_{c}$, which do not occur at all in CV and form only in 6.7\% of all cases (2/30) in CVi. In CVi we also observe a few other stars with the low-cost agent in the center (13.3\%; 4/30).

21 Notably in treatments V, VV, Vi, CV, and CVi, all stars formed are (Bayesian) Nash networks. In particular, in VV the observed 'other' stars with a highvalue agent in the center $\left(\operatorname{star}_{v}\right)$ and in CVi the 'other' stars with the low-cost agent in the center ( $\left.\operatorname{star}_{C}\right)$ are all minimally sponsored. In treatment C, 3 of the 5 observed $\operatorname{star}_{C}$ networks are minimally sponsored and, hence, Nash. In Ci and B none of the few observed other stars is a (Bayesian) Nash network. 22 The positive correlation in treatment $\mathrm{Ci}$ is due to the fact that up to round 16 no stars at all are formed whereas from round 17 onwards between 1 and 3 stars are formed in each round. In the other treatments with a significantly positive correlation coefficient the increase in the number of stars over time exhibits a much stronger pattern.
} 
$\operatorname{PSS}_{v} \ldots$ periphery sponsored star with $v$ in the center; $\operatorname{CSS}_{c} \ldots$ center sponsored star with $c$ in the center; $\operatorname{star}_{v} \ldots$ any star with $v$ in the center (not $\operatorname{PSS}_{v}$ ); $\operatorname{star}_{c} \ldots$ any star with $c$ in the center (not $\operatorname{CSS}_{c}$ ); other star ... any other star; other networks ... any other network
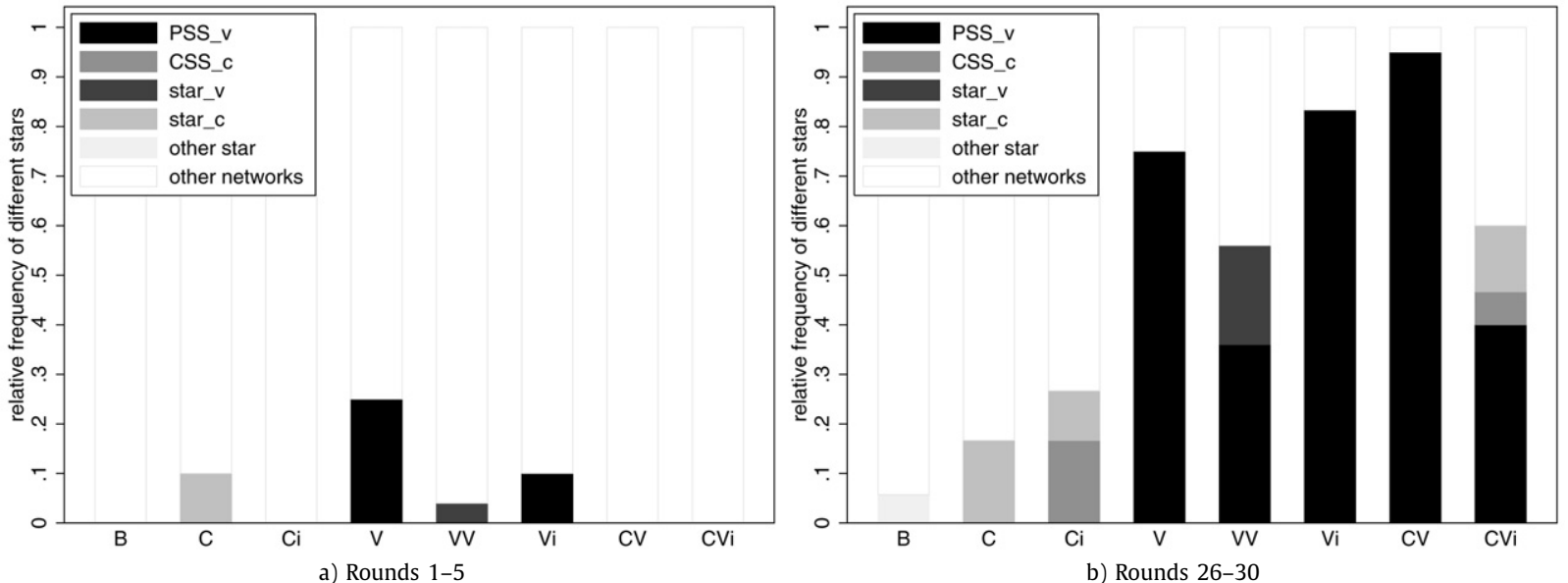

Fig. 7. Frequency of stars in the first five rounds and the last five rounds.

Network centrality. Individual centrality is a measure that is often employed in sociological studies to analyze how central (strong, important) agents' positions are in a network regarding, e.g., communication flow, interaction possibilities, or power. ${ }^{23}$ One can build upon the individual centrality measures to construct a measure of centrality for the whole network. While various definitions are possible, we use the so called 'degree-centrality' measure, which is defined as the sum of the differences in degrees between the most central agent and all other agents. Formally,

$$
\operatorname{cent}(g)=\frac{1}{20} \sum_{i \in N}\left[\max _{j \in N} \operatorname{deg}_{j}(g)-\operatorname{deg}_{i}(g)\right] .
$$

Important properties of degree-centrality (or other measures of centrality proposed in the literature) are that it is bounded and, given our normalization, 0 for 'even' networks such as the empty, complete, and wheel networks, and 1 if and only if the network is a star. Increasing centrality can be interpreted as a movement towards star or star-like networks. We are, therefore, particularly interested if the centrality measure increases over time and whether it approaches 1 .

Fig. 8 depicts the development of average centrality in all eight treatments over rounds (in blocks of 5 rounds). It shows that in early rounds the centrality of networks is very similar across treatments, with the exception of treatment V. Indeed, a Kruskal-Wallis equality of populations rank test does not reject the hypothesis that the centrality measures across the first five rounds are the same in all treatments $(p=0.6234)$. The development of centrality differs strongly across treatments, however.

For treatments V, Vi, VV, CV, and CVi centrality clearly shows an upward trend. To a smaller extent this also holds for Ci. In contrast, in B and C no such tendency is observed. Restricting attention to the average centrality in the last five rounds, a Kruskal-Wallis test rejects the hypothesis that centrality is the same in all treatments $(p=0.0051){ }^{24}$

To test whether network centrality approaches 1 we conducted Wilcoxon signed-rank tests based on the network centrality averaged across the last five rounds. The null hypothesis is that network centrality is equal to 1 . We reject this hypothesis for treatments B $(p=0.0178), \mathrm{C}(p=0.0277)$, and $\mathrm{Ci}(p=0.0350)$. The hypothesis is rejected only marginally for VV and CVi $(p=0.0523$ and $p=0.0897)$. In the other treatments, i.e., $\mathrm{V}, \mathrm{Vi}$, and $\mathrm{CV}$, we cannot reject the hypothesis $(p \geqslant 0.3173$; all tests two-sided). Hence, our earlier conclusions regarding star formation in the different treatments are corroborated.

\subsection{How efficient are the observed networks?}

The prevalence of $\mathrm{PSS}_{v}$ in CV indicates that the formation of stars does not necessarily lead to efficient outcomes. In this treatment the $\operatorname{CSS}_{c}$ network would be efficient but is never observed. Fig. 9 shows the development of the relative

\footnotetext{
23 See, e.g., Freeman (1979) and Wasserman and Faust (1994) for detailed discussions of centrality measures.

24 Pairwise comparisons using Mann-Whitney tests show that equality of centrality in B and C cannot be rejected ( $p=0.2827$; two-sided test) and that centrality in $\mathrm{V}, \mathrm{Vi}, \mathrm{VVi}, \mathrm{CV}$, and $\mathrm{CVi}$ is higher than in B and C (all comparisons at the 5 percent significance level, except for C vs. V and C vs. VV where $p=0.0842$ and $p=0.0996$, respectively; two-sided tests).
} 


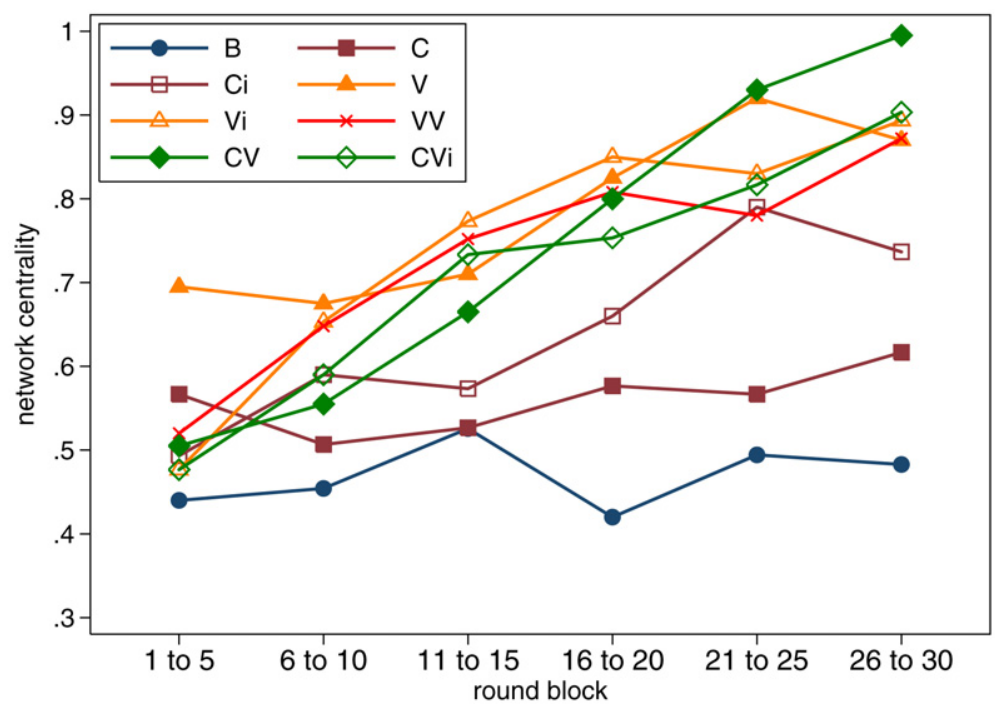

Fig. 8. Development of centrality over time.

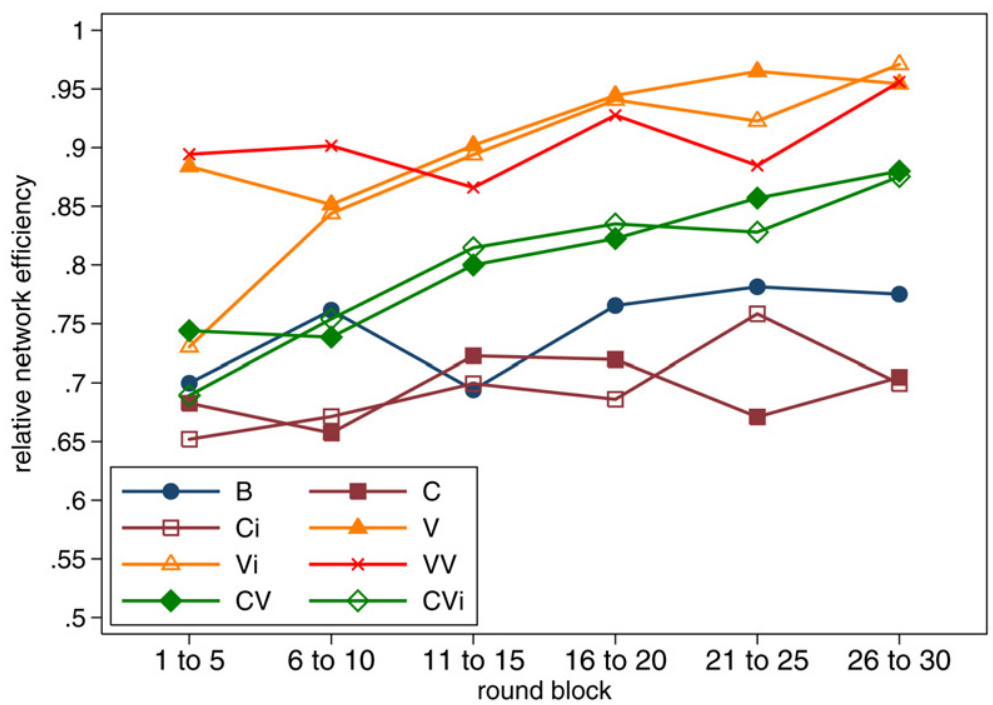

Fig. 9. Development of efficiency over time.

efficiency of observed networks for the different treatments aggregated over blocks of five rounds. Clearly, efficiency in early rounds (rounds 1-5) is similar across treatments (with the notable exceptions of V and VV where more efficient networks are formed). ${ }^{25}$ Fig. 9 shows that relative efficiencies in treatments V, VV, and VVi increase and converge to a common level. At a somewhat lower level a similar pattern holds for treatments CV and CVi. Over time the lowest efficiency levels occur in treatments $\mathrm{C}$ and $\mathrm{Ci}$, with $\mathrm{B}$ ending up between the latter and $\mathrm{CV}$ and CVi. Using data of only the last five rounds, a Kruskal-Wallis test clearly rejects the hypothesis that the efficiency levels for the eight treatments are the same $\left(\chi_{(7)}^{2}=24.728, p=0.0008\right.$, two-sided test $) .^{26}$

\footnotetext{
25 This impression is corroborated by a Kruskal-Wallis test for equality of populations between all treatments except $\mathrm{V}$ and $\mathrm{VV}\left(\chi_{(5)}^{2}=4.575, p=0.4699\right.$, two-sided test). Mann-Whitney tests comparing (pairwise) early-round efficiency in V and VV with other treatments shows significant differences in all cases at least at the 5 percent level, except for $\mathrm{V}$ and $\mathrm{VV}$ vs. Vi where the difference is only marginally significant $(p=0.0550$ and $p=0.0679$, respectively; all tests two-sided). The difference between $\mathrm{V}$ and $\mathrm{VV}$ is statistically insignificant ( $p=0.8065$ ).

${ }^{26}$ In the last five rounds, pairwise comparisons show that efficiency levels are not significantly different for treatments B, C, Ci ( $p \geqslant 0.1985$; two-sided). Efficiency levels in each of these treatments are significantly lower than in CV and CVi ( $p \leqslant 0.0430$; one-sided), which are not different from each other $(p=0.3367$; two-sided). Efficiency in Vi is significantly larger than in CV and CVi ( $p<0.0297$; one-sided). This also holds when comparing VV with CVi $(p=0.0268)$. Efficiency levels between V, Vi and VV, between V, CV and CVi, and between VV and CV do not significantly differ ( $p \leqslant 0.1231$; two-sided). All tests are Mann-Whitney tests.
} 


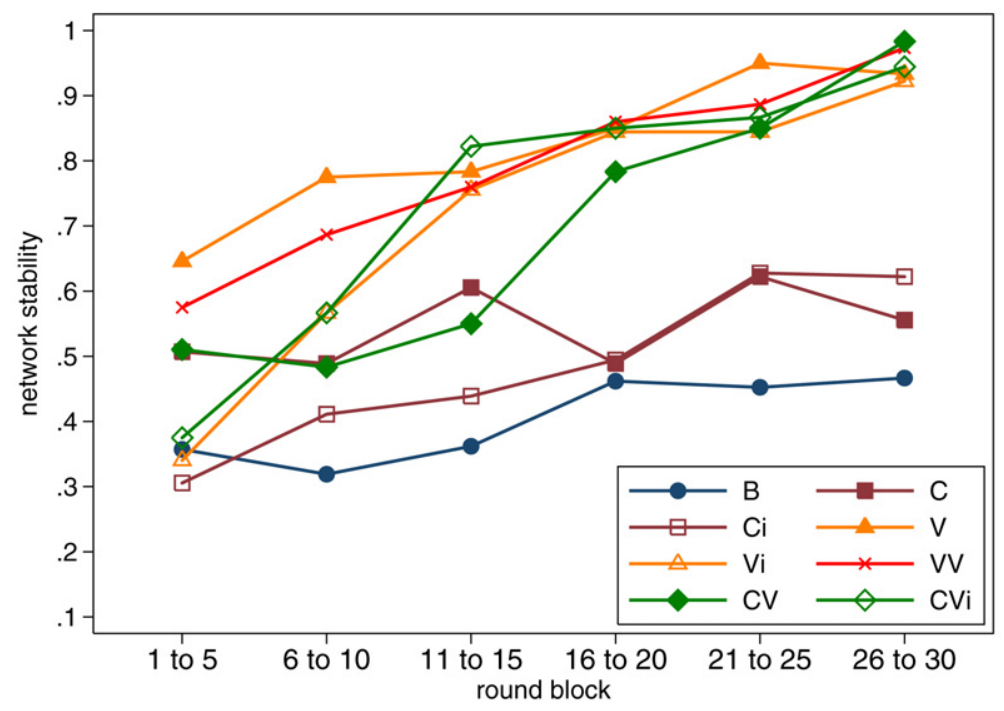

Fig. 10. Development of network stability over time.

In summary, observed network efficiency in the different treatments parallels the results regarding star formation. Efficiency levels are lowest in treatments where no high-value agent is present. In treatments where a high-value and a low-cost agent are present efficiency levels tend to be lower than in treatments with only high-value agents.

\subsection{How stable are the observed networks?}

We define the relative stability of a network in round $t$ as the number of agents who do not change any links from round $t-1$ to round $t$ divided by the total number of agents. Hence, if no agent changes a link, relative stability is 1 while it is 0 if all agents change at least one link.

Fig. 10 depicts the development of network stability over time for all treatments (in blocks of five rounds). There is a clear separation between treatments with and without at least one high-value agent. In the last few rounds, networks in treatments $\mathrm{CV}, \mathrm{CVi}, \mathrm{V}, \mathrm{Vi}$, and $\mathrm{VV}$ approach full stability. On average, the stability measure for these treatments is between 0.922 (in $\mathrm{Vi}$ ) and 0.983 (in CV). So, roughly speaking, towards the end of the experiment at most one person changes her behavior every other round. Relative stability is not significantly different from 1 for treatments $\mathrm{V}, \mathrm{Vi}, \mathrm{VV}$, and CV $(p \geqslant 0.1590$; two-sided Wilcoxon signed-rank test). In treatment CVi relative stability is also close to 1 but statistically marginally different from it $(p \geqslant 0.0516$; two-sided Wilcoxon signed-rank test), indicating that network formation did not completely settle down.

In the treatments without a high-value agent, networks remain unstable over time and there is no tendency towards stability. In the last five rounds, average relative stability is between 0.467 (in B) and 0.622 (in Ci), meaning that on average between 2.3 and 3.2 agents change behavior in each round. In the last five rounds, stability does not significantly differ across treatments $\mathrm{B}, \mathrm{C}$, and $\mathrm{Ci}\left(\chi_{(2)}^{2}=3.018, p=0.2211\right.$; Kruskal-Wallis test, two-sided). Mann-Whitney tests show that compared to the treatments with a high-value agent, stability is significantly smaller (pair-wise comparisons: $p<0.05$ in all cases except Ci vs. V where $p=0.0521$; two-sided tests).

In summary, network stability strongly improves in the presence of at least one high-value agent and networks reach (almost) full stability at the end of the experiment. In stark contrast, networks remain unstable in treatments without a high-value agent.

\subsection{Explaining treatment differences: Individual linking behavior}

The observed network structures are ultimately the outcome of individual linking decisions. To understand why star formation occurs less frequently with a low-cost agent than with a high-value agent-unlike the theoretical predictions-we estimate a structural model of linking behavior, based on noisy best-response dynamics and inequality averse preferences à la Fehr and Schmidt (1999).

Consider player $i$ in period $t>1$ and denote the network that the others formed in period $t-1$ by $g_{-i}^{t-1}$. The idea of noisy best responses is that the probability that player $i$ selects the link vector $g_{i}^{t}$ in period $t$ is proportional to the material payoffs associated with $g_{i}^{t}$ :

$$
\operatorname{Prob}\left(g_{i}^{t}\right)=\frac{e^{\lambda \pi_{i}\left(g_{i}^{t} \oplus g_{-i}^{t-1}\right)}}{\sum_{h_{i}^{t} \in \mathcal{G}_{i}} e^{\lambda \pi_{i}\left(h_{i}^{t} \oplus g_{-i}^{t-1}\right)}} .
$$


Table 3

Maximum-likelihood estimates.

\begin{tabular}{|c|c|c|c|c|c|}
\hline & \multicolumn{5}{|l|}{ All treatments } \\
\hline & $\lambda$ & $\alpha$ & $\beta$ & $\log L$ & \# Obs. \\
\hline Periods 2-16 & $2.59^{* *}(0.27)$ & $2.78^{* *}(0.39)$ & $-0.27(0.14)$ & -2.238 & 3960 \\
\hline Periods $16-30$ & $6.12^{* *}(0.30)$ & $1.69^{* *}(0.14)$ & $0.14^{* *}(0.05)$ & -1.490 & 3960 \\
\hline All periods & $4.13^{* *}(0.29)$ & $2.04^{* *}(0.21)$ & $0.05(0.06)$ & -1.911 & 7656 \\
\hline
\end{tabular}

Note: Standard errors are shown in parentheses. The reported log-likelihood is per observation.

${ }^{* *}$ Significance at the $1 \%$ level.

Here the "rationality" parameter, $\lambda$, measures how sensitive players are with respect to payoffs. For $\lambda=0$, they act randomly and pick any of the $\left(\left|\mathcal{G}_{i}\right|=32\right)$ available linking vectors with equal probability. At the other extreme, when $\lambda$ tends to infinity, players' behavior approaches perfect payoff maximization, i.e. the linking vector with the highest payoff is chosen with probability 1 . For in between values of $\lambda$, all linking vectors have some chance of being chosen. In other words, "mistakes" are possible although the likelihood of a certain mistake is inversely proportional to its severity.

The next step is to "dress up" the model with inequality aversion. The choice of linking vector $g_{i}^{t}$ by player $i$ in period $t$ not only determines her own material payoff but also those of others, $\pi_{j}\left(g_{i}^{t} \oplus g_{-i}^{t-1}\right)$ for $j \neq i$. We use the Fehr-Schmidt model in (5) to determine player $i$ 's utility given the profile of material payoffs, ${ }^{27}$ and the choice probabilities become

$$
\operatorname{Prob}\left(g_{i}^{t}\right)=\frac{e^{\lambda u_{i}\left(g_{i}^{t} \oplus g_{-i}^{t-1}\right)}}{\sum_{h_{i}^{t} \in \mathcal{G}_{i}} e^{\lambda u_{i}\left(h_{i}^{t} \oplus g_{-i}^{t-1}\right)}} .
$$

The best response aspect of the model represents some kind of "stability": if player $i$ has a set of links available that yield higher utility than her existing ones, the model predicts that she is likely to change her linking choices and the network would not be stable.

We apply the model in (7) to the pooled data from the complete and incomplete information treatments. We separately estimate the model for the first half (periods 2-16) and second half (periods 16-30) of the experiment to correct for possible learning effects. The results are reported in Table 3. Note that there are clear signs of learning as the estimated $\lambda$ is significantly higher in later periods, indicating that behavior becomes less noisy or more rational over time.

Of special interest are the estimated values of the envy and guilt parameters $\alpha$ and $\beta$. Recall from Section 2.4 that for certain $(\alpha, \beta)$ combinations, some of the equilibrium networks break down in treatments with a low-cost agent. Table 3 shows that in all periods, the maximum-likelihood estimates of $\alpha$ are high while those for $\beta$ are small. Indeed, pooling across periods the estimated envy parameter is 2 while the estimated guilt parameter is not substantially or significantly different from 0 .

To see how this affects star formation in the different treatments, consider the most obvious networks in the highvalue and low-cost treatments: the periphery-sponsored star PSS $_{v}$ in treatment V and the center-sponsored star CSS in $_{c}$ treatment $\mathrm{C}$. In the $\mathrm{PSS}_{v}$ network, the peripheral normal agents who support the links have material payoffs equal to 56 while the center's material payoffs are 80. If a peripheral agent were to remove her link (e.g., because she is envious of the center), her material payoffs would fall to 0 , while other peripheral agents would still earn 44 and the center 64 . In other words, with an estimated envy (guilt) parameter $\alpha=2(\beta=0)$, removing a link would reduce a peripheral agent's utility from approximately 46 to -90 .

Now consider $\mathrm{CSS}_{c}$, where the material payoffs of the low-cost agent in the center are 45 while the peripheral agents' material payoffs are 64, which translates into a utility of 7 for the center (using $\alpha=2$ ). If the low-cost agent instead supported 2 links (and these are the only links) then her material payoff is 18, three periphery agents earn nothing while two others earn 28. Ignoring any guilt $(\beta=0)$, this translates into a higher utility of 10 for the low-cost agent. More generally, the low-cost agent's utilities of making $0,1,2,3,4$ or 5 links (assuming these are the only links) are $0,6.2,10,11.4,10.4$ and 7, respectively. Under noisy best response dynamics, these utilities imply that the low-cost agent most often chooses to

$\overline{27}$ In other words, player $i$ 's utility of choosing linking vector $g_{i}^{t}$ becomes

$$
\begin{aligned}
u_{i}\left(g_{i}^{t} \oplus g_{-i}^{t-1}\right)= & \pi_{i}\left(g_{i}^{t} \oplus g_{-i}^{t-1}\right) \\
& -\frac{\alpha_{i}}{n-1} \sum_{j} \max \left\{\pi_{j}\left(g_{i}^{t} \oplus g_{-i}^{t-1}\right)-\pi_{i}\left(g_{i}^{t} \oplus g_{-i}^{t-1}\right), 0\right\} \\
& -\frac{\beta_{i}}{n-1} \sum_{j} \max \left\{\pi_{i}\left(g_{i}^{t} \oplus g_{-i}^{t-1}\right)-\pi_{j}\left(g_{i}^{t} \oplus g_{-i}^{t-1}\right), 0\right\},
\end{aligned}
$$

where $\pi_{j}\left(g_{i}^{t} \oplus g_{-i}^{t-1}\right)$ is player $i$ 's estimate of player $j$ 's payoff given that player $i$ chooses linking vector $g_{i}^{t}$ and given the network $g_{-i}^{t-1}$ formed by others in period $t-1$. 
make 3 links, but that constellations where she makes 2 or 4 links are also quite likely. ${ }^{28}$ In particular, these results explain the difficulties in coordinating on the intuitive outcome with a low-cost agent, i.e. the $\operatorname{CSS}_{c}$ network.

\section{Conclusion}

Casual empiricism suggests that individual differences may facilitate the creation of uneven, hierarchal structures such as star networks. Some people have lower opportunity costs or a taste for networking, while others may possess skills or information that is relatively more scarce. We conjectured that the presence of these "special" individuals facilitates the creation of star-type networks because it reduces the coordination problem and makes the resulting payoff inequalities more acceptable.

This paper reports the first experimental study on endogenous network formation with heterogeneous agents. We consider several implementations of individual heterogeneity (and allow agents' types to be private information or publicly known), and provide a complete characterization of the set of (Bayesian) Nash equilibria for all treatments. Specifically, we introduce subjects who have a higher value to others (high-value treatments) and subjects who have lower linking costs then others (low-cost treatments). In our baseline treatment with symmetric agents almost no stars are formed. In contrast the introduction of different types of agents has a dramatic impact on linking behavior and observed networks: stars are prevalent in all treatments with high-value agents, with this agent typically being the center of the star. Stars are not born, however, but grow over time. Early on in the experiment almost no stars are formed, which suggests that none of the star networks is an obvious or focal outcome. But towards the end of the experiment, stars are the most prevalent architecture in treatments with a high-value agent. Interestingly, introducing a low-cost agent does not substantially foster star formation. These findings are underlined by the evolutions of network centrality, network efficiency, and network stability, which all indicate a strong trend towards stable star networks only when a high-value agent is present.

The effects of incomplete information depend on the types of agents in the network. With an unknown high-value agent, subjects are quick to find this agent and form a periphery-sponsored star. In this case, network formation is not significantly different from the complete-information case. Likewise, with a low-cost agent, incomplete information slightly raises the occurrence of stars with the low-cost agent at the center, but again the differences are not statistically different from the complete-information case. When both types of agents are present, incomplete information aggravates the coordination problem and significantly fewer stars are formed. Moreover, a significant fraction of the observed stars do not have the high-value agent at the center unlike the complete information case where all stars are of this type.

While the introduction of a high-value agent strongly enhances the likelihood of star formation, this is not the case for the introduction of a "networker" with lower linking costs. Again this suggests it is not simply "focalness" that determines the empirical likelihood of star formation. Intuitively, an important difference is that with a low-cost agent the focal network is a center-sponsored star where the center may become envious towards those she sponsors. In contrast, the focal network with a high-value agent is the periphery-sponsored star where the center has no opportunity to deviate by removing links and peripheral agents would hurt themselves more than others when removing their links.

In order to formalize this intuition and explain the observed treatment effects, we apply a structural econometric model based on best-response dynamics and inequality-averse preferences. The maximum-likelihood estimates of the underlying structural parameters reveal why stars form in treatments with a high-value agent but not in treatments with a low-cost agent. In particular, the estimated envy parameter is high enough such that the periphery-sponsored star in the high-value treatment is a strict Nash network, but the center-sponsored star in the low-cost treatment is not.

\section{Appendix A. Proofs}

In Lemma 5 below we characterize for each incomplete information treatment the expected payoff of each agent in each possible network. We next use this characterization to show in Proposition 6 below that all (Bayesian) Nash networks in all treatments are either minimally connected or empty. With the help of Lemma 5 and Proposition 6 we then prove Propositions 1, 2 and 3 .

The decay function used in our experiments can be expressed as $\Phi\left(v_{i}, d\right)=v_{i}\left(v^{\circ}(d) / 16\right)$, with $v$ given by $\left(v^{\circ}(1)\right.$, $v^{\circ}(2)$, $\left.v^{\circ}(3), v^{\circ}(4), v^{\circ}(5), v^{\circ}(\infty)\right)=(16,12,9,7,5,0)$, see Table $1 \mathrm{~b}$. Hence, the benefit from accessing a normal value agent at distance $d$ is $\Phi(16, d)=v^{\circ}(d)$, while the benefit from accessing a high-value agent at the same distance is $\Phi(32, d)=$ $2 v^{\circ}(d)$. The following lemma characterizes an agent's expected payoff in the incomplete information treatments.

Lemma 5. Given a cost/value profile $\theta$, the expected payoffs of different types of agents in the incomplete information treatments are:

1. For $i \neq i_{v}$ in Vi and CVi: $E_{i} u_{i}\left(g ; \theta_{i}\right)=\frac{6}{5} \sum_{j \neq i} \nu^{\circ}(d(i, j ; g))-\mu_{i}(g) c$.

2. For $i_{v}$ in Vi and CVi and for any $i$ in $C i: E_{i} u_{i}\left(g ; \theta_{i}\right)=\sum_{j \neq i} \nu^{\circ}(d(i, j ; g))-\mu_{i}(g) c$.

\footnotetext{
28 In the experiments, the low-cost agent made 2.2 links on average. Of course, in the experiments also the normal agents made links ( 0.75 on average), which alters the utility comparisons mentioned in the main text.
} 
Proof. Note from (2) that agent $i$ 's payoff is independent of the linking costs incurred by other agents $j \neq i$. Agent $i$ 's expected payoff therefore depends only on her beliefs about others' values.

1. Given the realized profile $\theta$, any agent $i \neq i_{v}$ in treatments Vi and CVi knows that any of the other agents is equally likely to be the unique high-value agent. For each $k \neq i$, let $w^{k}=\left(w_{1}^{k}, \ldots, w_{n}^{k}\right)$ be one of the five possible value profiles from $i$ 's perspective, with $w_{k}^{k}=32$ and $w_{j}^{k}=16$ for $j \neq k$, and $p_{i}^{\theta_{i}}\left[w^{k}\right]=\frac{1}{5}$. Agent $i$ 's expected payoff, given the network $g$, is

$$
\begin{aligned}
E_{i} u_{i}\left(g ; \theta_{i}\right) & =\sum_{k \neq i} p_{i}^{\theta_{i}}\left[w^{k}\right] \sum_{j \neq i} \Phi\left(w_{j}^{k}, d(i, j ; g)\right)-\mu_{i}(g) c_{i}(\theta) \\
& =\sum_{k \neq i} \frac{1}{5} \sum_{j \neq i} \frac{w_{j}^{k}}{16} v^{\circ}(d(i, j ; g))-\mu_{i}(g) c_{i}=\frac{1}{80} \sum_{j \neq i} v^{\circ}(d(i, j ; g)) \sum_{k \neq i} w_{j}^{k}-\mu_{i}(g) c_{i} \\
& =\frac{1}{80} \sum_{j \neq i} v^{\circ}(d(i, j ; g)) 96-\mu_{i}(g) c_{i}=\frac{6}{5} \sum_{j \neq i} v^{\circ}(d(i, j ; g))-\mu_{i}(g) c_{i} .
\end{aligned}
$$

2. Given the realized profile $\theta$, all agents in treatment $\mathrm{Ci}$ and agent $i_{v}$ in treatments $\mathrm{Vi}$ and $\mathrm{CVi}$ know the value of any other agent is 16 . Hence, for these agents the expected payoff, given the network $g$, is $E_{i} u_{i}\left(g ; \theta_{i}\right)=\sum_{j \neq i} \Phi(16, d(i, j ; g))-$ $\mu_{i}(g) c_{i}=\sum_{j \neq i} \nu^{\circ}(d(i, j ; g))-\mu_{i}(g) c_{i}$.

Proposition 6. With complete or incomplete information, (Bayesian) Nash networks are either minimally connected or empty.

Proof. It is easy to see that a (Bayesian) Nash network is minimally sponsored: an agent incurs costs but no additional benefits from making a link to another agent who already maintains this link. It is also straightforward to see that whenever a link is made between $i \neq j$, any other agent that does not access $i$ or $j$ is willing to sponsor a link with either of them. This implies that a (Bayesian) Nash network is either empty or connected. Next we show that such a network has no cycles. We do this by arguing that in any minimal cycle there is at least one agent who (weakly) benefits from removing a link from the cycle. Below we use the notation $i j$ to denote a link made by $i$ to $j$.

Treatments $B, V, C$ : Let $g$ be a minimally sponsored network with a shortest circle of length $k \geqslant 3$. It is easy to see that in any such circle at least one link must be sponsored by an agent of normal linking cost whose distance to the high-value agent (if present) does not change if she removes that link. Let $(1, \ldots, k, 1)$ denote such a circle and $1 k$ be the corresponding link. Recall that the linking cost of (normal) agent 1 is $c=24$.

If 1 removes the link $1 k$, yielding the network $g_{-1 k}$, the distances between her and other agents may increase, which lowers agent 1 's benefits. It is convenient to define $\Delta v^{\circ}(d, \Delta d)=v^{\circ}(d)-v^{\circ}(d+\Delta d)$ as the loss in benefits that result when the distance $d$ to a normal-value agent increases by $\Delta d$. The loss function $\Delta v^{\circ}(d, \Delta d)$ is decreasing in $d$ and increasing in $\Delta d$. For each $j$, let $\Delta_{i k} d(i, j ; g)=d\left(i, j ; g_{-i k}\right)-d(i, j ; g)$ denote the increase in the distance between $i$ and $j$ when link $i k$ is removed from $g$, and let

$$
\begin{aligned}
\Delta_{k} \pi_{i}(g) & =\pi_{i}(g)-\pi_{i}\left(g_{-i k}\right) \\
& =c_{i}+\sum_{j \neq i} \frac{v_{j}}{16}\left[v^{\circ}(d(i, j ; g))-v^{\circ}\left(d\left(i, j ; g_{-i k}\right)\right)\right] \\
& =c_{i}-\sum_{j \neq i} \frac{v_{j}}{16} \Delta v^{\circ}\left(d(i, j ; g), \Delta_{i k} d(i, j ; g)\right)
\end{aligned}
$$

denote the corresponding change in agent $i$ 's payoff. When agent 1 removes the link $1 k$, the distances between herself and agents $\left\{2, \ldots,\left\lceil\frac{k+1}{2}\right\rceil\right\}$ remains the same, but the distances to all (normal-valued) other agents may increase. Her distance to any agent $j>k$ increases by at most $k-2$, hence $\Delta v^{\circ}(2, k-2)$ is the maximum possible decrease in benefits for $i$ obtained from a normal agent $j$ with whom $i$ did not have a direct link in $g$. The change of agent 1's payoff, when she removes the link $1 k$, is therefore bounded by

$$
\Delta_{k} \pi_{1}(g) \geqslant 24-\sum_{j=\left\lceil\frac{k+3}{2}\right\rceil}^{k}\left[v^{\circ}(j-1)-v^{\circ}(k-j+1)\right]-(n-k) \Delta v^{\circ}(2, k-2) .
$$

For $k=3,4,5,6$ this bound is strictly positive $\left(\Delta_{3} \pi_{1}(g) \geqslant 11, \Delta_{4} \pi_{1}(g) \geqslant 7, \Delta_{5} \pi_{1}(g) \geqslant 5, \Delta_{6} \pi_{1}(g) \geqslant 8\right)$, which implies that agent 1 always benefits from removing the link $1 k$.

Treatment CV: In a Nash network the low-cost agent $i_{c}$ will always be linked with the high-value agent $i_{v}$. Indeed, $i_{c}$ is willing to sponsor the link with $i_{v}$ in any minimally sponsored $g$ as the loss from removing such a link is at least $2 \Delta v^{\circ}\left(d\left(i_{c}, i_{v} ; g\right), \Delta d_{i_{c}, i_{v}}\left(i_{c}, i_{v} ; g\right)\right) \geqslant 2 \Delta v^{\circ}(1,1)=8$, which exceeds $i_{c}$ 's linking cost of 7 . Since $i_{c}$ and $i_{v}$ are linked, in any circle of length $k \geqslant 4$ at least one link is sponsored by a normal agent whose distance to the high-value agent (if present) 
does not change if she removes that link. The same conclusion holds for a circle of length $k=3$ where either $i_{c}$ or $i_{v}$ is not part of the circle. In both situations, the proof that applied to the $\mathrm{B}, \mathrm{V}$, and $\mathrm{C}$ cases can be replicated.

Finally, suppose that both $i_{c}$ and $i_{v}$ are part a cycle of length $k=3$, including a normal agent $i$ who sponsors the link with $i_{v}$ (if link $i_{v} i$ is sponsored by $i_{v}$, then the proof above applies). By removing this link agent $i$ gains at least $\Delta_{i_{v}} \pi_{i}(g) \geqslant 24-(32 / 16)\left[v^{\circ}(2)-v^{\circ}(1)\right]-3 \Delta v^{\circ}(2,1)=7$.

Treatment $V V$ : When the distance to a high-value agent increases from $d$ to $d+\Delta d$ an agent looses a benefit of $2 \Delta v^{\circ}(d, \Delta d)$. Let $g$ be a minimally sponsored network with a shortest cycle $(1,2, \ldots, k, 1)$ of length $k \geqslant 3$. The precise gain by removing a link from this cycle depends on the location of $i_{v}$ and $j_{v}$. Suppose that $k=6$ and let $1 k$ link two normal agents. By removing this link agent 1 gains at least $\Delta_{k} \pi_{1}(g) \geqslant 24-\left(2 \Delta \nu^{\circ}(2,2)+\Delta \nu^{\circ}(1,4)\right)=3$. Suppose now that $k=5$. If both $i_{v}$ and $j_{v}$ are part of the cycle then it contains a link between two normal agents; let it be $1 k$. By removing it agent 1 gains at least $\Delta_{k} \pi_{1}(g) \geqslant 24-\left(2 \Delta \nu^{\circ}(2,1)+\Delta v^{\circ}(1,3)\right)-\Delta v^{\circ}(2,3)=2$. If, however, one-let it be $i_{v}$-is not part of the cycle then a link $i j$ in the cycle can be removed without increasing the distance of $i$ to $i_{v}$; let it be $1 k$. By removing it agent 1 gains at least $\Delta_{k} \pi_{1}(g) \geqslant 24-\left(\Delta v^{\circ}(2,1)+2 \Delta v^{\circ}(1,3)\right)=3$. Suppose now that $k=4$. In this cycle at least one link can be removed increasing a distance to at most one agent not part of the cycle; let it be $1 k$. By removing it agent 1 gains at least $\Delta_{k} \pi_{1}(g) \geqslant 24-2 \Delta v^{\circ}(1,2)-2 \Delta v^{\circ}(2,2)=0$. Suppose finally that $k=3$ with a link $1 k$. By removing it agent 1 gains at least $\Delta_{k} \pi_{1}(g) \geqslant 24-2 \Delta \nu^{\circ}(1,1)-\left(2 \Delta \nu^{\circ}(2,1)+\Delta \nu^{\circ}(2,1)+\Delta \nu^{\circ}(2,1)\right)=4$.

Treatments $\mathrm{Vi}, \mathrm{Ci}, \mathrm{CVi}$ : Let $\theta$ be a feasible allocation of types. Lemma 5 implies that, compared to the complete information case, normal agents' benefits are multiplied by factor 6/5 in treatments Vi and CVi while they are unchanged in treatment Ci. Define $\Delta_{k} E_{i} u_{i}\left(g ; \theta_{i}\right)=E_{i} u_{i}\left(g ; \theta_{i}\right)-E_{i} u_{i}\left(g_{-i k} ; \theta_{i}\right)$. Again, let $(1, \ldots, k, 1)$ be the shortest cycle in $g$ and let agent 1 satisfy the assumptions above. Following the steps of the proof for treatment $\mathrm{B}$, inequality (8) becomes

$$
\Delta_{k} E_{1} u_{1}\left(g ; \theta_{1}\right) \geqslant 24-\frac{6}{5} \sum_{j=\left\lceil\frac{k}{2}\right\rceil+1}^{k}\left[v^{\circ}(j-1)-v^{\circ}(k-j+1)\right]-\frac{6}{5}(n-k) \Delta v^{\circ}(2, k-2)
$$

for treatments Vi and CVi. The corresponding bounds $\left(\Delta_{3} E_{1} u_{1}\left(g ; \theta_{1}\right) \geqslant 8.4, \Delta_{4} E_{1} u_{1}\left(g ; \theta_{1}\right) \geqslant 3.6, \Delta_{5} E_{1} u_{1}\left(g ; \theta_{1}\right) \geqslant 1.2\right.$, $\left.\Delta_{6} E_{1} u_{1}\left(g ; \theta_{1}\right) \geqslant 4.8\right)$ are all strictly positive. For treatment $\mathrm{Ci}$ the bounds found in the proof for treatment $\mathrm{B}$ apply.

Proof of Proposition 1. We leave it to the reader to verify that the listed networks are strict Nash (hereafter SNN). Note that the benefit to a normal cost agent for making a link with an isolated normal value agent is 16 and the cost is 24 . In a Nash network (hereafter NN) the normal cost agent therefore never links to a periphery normal agent.

Next consider two distinct periphery agents $i$ and $j$, each being the sponsor of their (only) link. Let $i$ be linked to $i_{1}$ and $j$ be linked to $j_{1} \neq i_{1}$. If the network is Nash, agent $i$ must find linking to $i_{1}$ at least as beneficial as linking to $j_{1}$. If $j$ removes her link with $j_{1}$ then agent $i$ should find linking to $i_{1}$ strictly more beneficial than linking to $j_{1}$. But then agent $j$ should find linking to $i_{1}$ also strictly more beneficial than linking to $j_{1}$, which contradicts the assumption that the network is Nash. Thus in a Nash network all periphery agents who sponsor their only link are linked to the same central agent.

This provides some intuition for the predominance of periphery-sponsored stars among the NN. The rest of the proof proceeds by considering each treatment separately. Proposition 6 implies that, in a non-empty NN, each pair of agents is connected by a single path.

Treatment B: Let $g$ be a non-empty, minimally connected NN. The two agents $i$ and $j$ who are furthest apart in $g$ must be periphery and therefore sponsor their own link. They must therefore be linked to the same agent, which implies that 2 is the maximal distance between agents in $g$. The star network is the only minimally connected network with maximal distance of 2 . The only non-empty NN is therefore the periphery-sponsored star.

Treatment $V$ : Let $g$ be a SNN. Normal agents are willing to sponsor a link with an isolated $i_{v}$ regardless of other links in a network, so $g$ cannot be empty. Let $i$ and $j$ be the two periphery agents who are furthest apart in $g$. Either both sponsor their own link and $g$ is a star network, or one of them is $i_{v}$. Let, in the latter case, $\left(i_{v}, i_{1}, \ldots, i_{k}, j\right)$ be the shortest path between $i_{v}$ and $j$, spanning $k+2$ agents. If $k \geqslant 2$ then none of the (at most two) remaining agents is further than $k+2$ from each $i_{v}$ and $j$. Each remaining agent must thence be directly linked with one of $\left\{i_{1}, \ldots, i_{k}\right\}$. The reader can verify that agent $j$ (weakly) prefers to move her link regardless of how the remaining agents are linked whenever $k \geqslant 2$. Therefore, $k=1$ in a SNN $g$, the maximal distance is 2 , and $g$ is a star network in which all normal periphery agents sponsor their link.

Treatment $C$ : Let $g$ be a SNN. Agent $i_{c}$ is willing to sponsor a link with any agent $i$ who, in absence of such link, would be more than 2 links away or isolated. Hence $g$ cannot be empty. Let $i$ and $j$ be the two periphery agents who are furthest apart in $g$. If both sponsor their own link then $g$ is a star network. If the center of $g$ is a normal agent then $g$ is peripherysponsored.

If $d(i, j) \geqslant 3$ then agent $i_{c}$ must be sponsoring the link with either $i$ or $j$, say with $i$. As $d\left(i_{c}, j\right) \leqslant 2$ for each $j$ and $d\left(i_{c}, i\right)=1$ then $d(i, j)=3$ with a path $\left(i, i_{c}, j_{1}, j\right)$. All other periphery agents sponsoring their own link must, as we argued above, also be linked with $j_{1}$. Finally, only agent $i_{c}$ would sponsor the links with agents not linked to $j_{1}$. This defines the 
linked star $\mathrm{LS}_{i_{c} j_{1}}^{p}$. It is easy to verify that if $p \geqslant 2$ all other periphery agents would prefer linking to $i_{c}$. The star networks PSS and $\mathrm{MSS}_{c}$, and the linked star $\mathrm{LS}_{i_{c} j_{1}}^{1}$ where $i_{c}$ sponsors the link with $i_{j}$, are the only SNN.

Treatment $C V$ : Let $g$ be a SNN. Agent $i_{c}$ is willing to sponsor a link to $i_{v}$ regardless of other links. Therefore, a star network with a normal agent in the center cannot be NN. If $g$ is not a star then it is a linked star with agent $i_{c}$ in one center, see the proof for treatment $\mathrm{C}$. If $i_{v}$ is a periphery agent linked with $i_{c}$ then other periphery agents (weakly) prefer to link with $i_{c}$, see the proof for treatment $\mathrm{V}$. Agent $i_{v}$ must therefore be in the other center of the linked star. It is easy to verify that $g$ is SNN if either all or less than three periphery normal agents are linked with $i_{c}$, and eliminate all remaining candidates for strict Nash networks but the $\mathrm{MSS}_{c}$, any $\mathrm{LS}_{c v}^{2}$, the $\mathrm{LS}_{c v}^{1}$ where $i_{c}$ sponsors the link with $i_{v}$, and $\mathrm{LS}_{c v}^{0}=\mathrm{PSS}_{v}$.

Treatment VV: Let $g$ be a SNN and let $i_{v}$ and $j_{v}$ be the two high-value agents. Normal agents are willing to sponsor a link with an isolated $i_{v}$ regardless of other links in a network, so $g$ cannot be empty. Let $i$ and $j$ be the two periphery agents who are furthest apart in $g$. Either both sponsor their own link and $g$ is a star network, or at least one of them is high-value. Let, in the latter case, $\left(i_{v}, i_{1}, \ldots, i_{k}, j\right)$ be the shortest path between $i_{v}$ and $j$, spanning $k+2$ agents. If $k \geqslant 2$ then none of the (at most two) remaining agents is further than $k+2$ from each $i_{v}$ and $j$. Each remaining agent must thence be directly linked with one of $\left\{i_{1}, \ldots, i_{k}\right\}$. Assume first that $k \geqslant 3$. If $j_{v} \in\left\{i_{1}, i_{2}\right\}$ or $j_{v}$ is a periphery directly linked to $i_{2}$ then $j$ prefers to move her link $j k_{3}$ to form $j k_{1}$. If, on the other hand, $j_{v}=i_{3}$ or is a periphery directly linked to $i_{3}$ then the link $i_{1} i_{2}$ must be sponsored by $i_{1}$ (as $i_{2}$ would prefer to link to $i_{v}$ ) who, however, prefers to move it to form $i_{1} i_{3}$. This proves that $k$ cannot be larger than 2. Assume now that $k=2$. If $j_{v}=k_{1}$ then $j$ prefers to move her link $j i_{2}$ to $j i_{1}$. It must therefore be that either $j_{v}=i_{2}$ or $j_{v}$ is a periphery linked with $i_{2}$, and that $i_{1}$ sponsors both links $i_{1} i_{v}$ and $i_{1} i_{2}$. In the latter case, the network is strict Nash only when $i_{2}$ sponsors the link with $j_{v}$. The two thus characterized linked stars are therefore the only SNN other than the star networks in which all normal periphery agents sponsor the link with the center.

Proof of Proposition 2. Again, we leave it to the reader to verify that the listed networks are strict Bayesian Nash. The proof closely follows the steps in the proof of Proposition 1, using the expected payoffs characterized by Lemma 5. In particular, characterization of strict Bayesian Nash networks in treatment Vi follows the same steps as those for treatment B, and characterization of strict Bayesian Nash networks in treatments Ci and CVi follows those for treatment C.

Proof of Proposition 3. The sum of agents' payoffs in a group can be written as

$$
w(g)=\sum_{i, j \in N} \Phi\left(v_{j}, d(i, j ; g)\right)-\sum_{i \in N} \mu_{i}(g) c_{i} .
$$

The efficient network therefore maximizes the aggregate benefits while minimizing the linking costs, i.e. it minimizes the distances between agents using a minimal number of links. Since adding a link to connect any two components of the network increases total payoffs, the efficient network is either empty or connected. In the latter case, it has at least $n-1$ links. Of the networks with precisely $n-1$ links the star networks have minimal average distances. In addition, the $\mathrm{CSS}_{c}$ with the low-cost agent $i_{c}$ in the center minimizes the aggregate linking costs, while a MSS $v$ with the high-value agent $i_{v}$ in the center maximizes the aggregate benefits. One can verify that $w\left(\mathrm{CSS}_{c}\right)>w\left(\mathrm{MSS}_{v}\right)$ when both $i_{c}$ and $i_{v}$ are present. Hence, among the star networks, $\operatorname{CSS}_{c}$ is efficient whenever $i_{c}$ is present and MSS $_{v}$ are efficient when only $i_{v}$ is present. Any MSS is the efficient star network in B. In a star network each new link benefits only the two linked agents. Adding links to the $\mathrm{CSS}_{c}$ thus decreases total payoffs: each link costs 24 but adds only 4 to the payoff of one agent and at most 8 to the payoff of the other (if one agent is a high-value agent). Adding links to a MSS ${ }_{v}$ or MSS ${ }_{o}$ when $i_{c}$ is not present also decreases total payoffs. Finally, total payoffs decrease if links are removed from any MSS. The above-mentioned star networks are therefore the efficient networks.

Proof of Proposition 4. Define $[x]_{+}=\max \{x, 0\}$ and $|x|=\max \{x,-x\}$. For each player $i$ and each profile of earnings $\pi=$ $\left(\pi_{1}, \ldots, \pi_{n}\right)$ let $a_{i}(\pi)=\frac{1}{n-1} \sum_{j=1}^{n}\left[\pi_{j}-\pi_{i}\right]_{+}, b_{i}(\pi)=\frac{1}{n-1} \sum_{j=1}^{n}\left[\pi_{i}-\pi_{j}\right]_{+}$and $q_{i}(\pi)=a_{i}(\pi)+b_{i}(\pi)=\sum_{j=1}^{n}\left|\pi_{j}-\pi_{i}\right|$ be her average disadvantageous, advantageous and total inequities in $\pi$ and let her corresponding utility be given by $U_{i}(\pi)=$ $\pi_{i}-\alpha_{i} a_{i}(\pi)-\beta_{i} b_{i}(\pi)$. We refer to $a_{i}(\pi), b_{i}(\pi)$ and $q_{i}(\pi)$ as envy, guilt, and inequity, respectively.

We begin by showing that an inequity averse player $i$ (weakly) prefers a profile of earnings $\pi$ over another profile $\pi^{\prime}=\left(\pi_{1}^{\prime}, \ldots, \pi_{n}^{\prime}\right)$ whenever her earnings (weakly) decrease from $\pi$ to $\pi^{\prime}$, her envy increases, but these two changes are not compensated by a large decrease in her guilt. That is,

$$
U_{i}(\pi) \geqslant U_{i}\left(\pi^{\prime}\right) \text { whenever } \pi_{i}^{\prime} \leqslant \pi_{i}, a_{i}\left(\pi^{\prime}\right) \geqslant a_{i}(\pi) \text { and } q_{i}\left(\pi^{\prime}\right) \geqslant q_{i}(\pi)-\left(\pi_{i}-\pi_{i}^{\prime}\right),
$$

with the utility of $\pi$ strictly larger whenever any inequality on the right side is strict. To see this, let $\Delta \pi_{i}=\pi_{i}-\pi_{i}^{\prime} \geqslant 0$ be the change in $i$ 's earnings when moving from $\pi^{\prime}$ to $\pi$ and let $\Delta a_{i}, \Delta b_{i}$ and $\Delta q_{i}=\Delta a_{i}+\Delta b_{i}$ be the corresponding changes in her envy, guilt and inequity. Statement (9) is straightforward when $\beta_{i}=0$, while for $1>\beta>0$ it follows from 


$$
\begin{aligned}
U_{i}(\pi) & =\pi_{i}-\pi_{i}^{\prime}+\pi_{i}^{\prime}-\alpha_{i}\left[a_{i}(\pi)+a_{i}\left(\pi^{\prime}\right)-a_{i}\left(\pi^{\prime}\right)\right]-\beta_{i}\left[b_{i}(\pi)+b_{i}\left(\pi^{\prime}\right)-b_{i}\left(\pi^{\prime}\right)\right] \\
& =U\left(\pi_{i}^{\prime}\right)+\Delta \pi_{i}-\alpha_{i} \Delta a_{i}-\beta_{i} \Delta b_{i} \\
& \geqslant U\left(\pi_{i}^{\prime}\right)+\beta_{i} \Delta \pi_{i}-\beta_{i} \Delta a_{i}-\beta_{i} \Delta b_{i}=U\left(\pi_{i}^{\prime}\right)+\beta_{i}\left(\Delta \pi_{i}-\Delta q_{i}\right) \\
& \geqslant U_{i}\left(\pi^{\prime}\right) .
\end{aligned}
$$

One consequence of (9) is that an agent prefers profile $\pi$ over profile $\pi^{\prime}$ whenever she is the lowest earner in each but earns less and faces larger total inequity in the latter. This implies, for instance, that inequity averse agents are not willing to make any links in an otherwise empty network. Another consequence of (9) is that an agent is not willing to make an action that decreases others' joint earnings and decreases her own earnings more than those of the other agents, that is

$$
U_{i}(\pi) \geqslant U_{i}\left(\pi^{\prime}\right) \text { whenever } \Delta \pi_{i} \geqslant \Delta \pi_{j} \text { for all } j \text {, and } \sum_{j \neq i} \pi_{j} \geqslant \sum_{j \neq i} \pi_{j}^{\prime}
$$

with the utility of $\pi$ strictly larger whenever any inequality on the right side is strict. To see this, recall first that $[x]_{+} \geqslant[y]_{+}$ and $x-y \geqslant[x]_{+}-[y]_{+}$whenever $x \geqslant y$ and note that the conditions in (10) imply $\pi_{i}-\pi_{j} \geqslant \pi_{i}^{\prime}-\pi_{j}^{\prime}$. This further implies, first $\left[\pi_{j}-\pi_{i}\right]_{+}-\left[\pi_{j}^{\prime}-\pi_{i}^{\prime}\right]_{+} \leqslant 0$ and thus $\Delta a_{i} \leqslant 0 \leqslant \frac{1}{n-1} \sum_{j \neq i} \Delta \pi_{j}$, and second

$$
\begin{aligned}
q_{i}-q_{i}^{\prime} & =\Delta q_{i}=\Delta a_{i}+\Delta b_{i}=\Delta a_{i}+\frac{1}{n-1} \sum_{j \neq i}\left(\left[\pi_{i}-\pi_{j}\right]_{+}-\left[\pi_{i}^{\prime}-\pi_{j}^{\prime}\right]_{+}\right) \\
& \leqslant \Delta a_{i}+\frac{1}{n-1} \sum_{j \neq i}\left(\pi_{i}-\pi_{j}-\pi_{i}^{\prime}+\pi_{j}^{\prime}\right)=\frac{1}{n-1} \sum_{j \neq i} \Delta \pi_{i}-\frac{1}{n-1} \sum_{j \neq i} \Delta \pi_{j}+\Delta a_{i} \\
& \leqslant \Delta \pi_{i} .
\end{aligned}
$$

Finally, (9) implies $U_{i}(\pi)>U_{i}\left(\pi^{\prime}\right)$.

One consequence of (10) is that no agent would make a link that is already established, as this would decrease her own earning but would not change the earnings of other agents. Consequently, an equilibrium network is minimal. Another consequence is that no periphery agent is willing to remove its only link from a connected network.

Observations (9) and (10) can now be used, following the steps below, to conclude that no inequity averse normal-cost agent wants to change her linking in any star or linked star listed in Proposition 1, except in those identified in Proposition 4. We leave it to the reader to verify that the following holds in all relevant cases. First, by removing or moving its only link a periphery normal-cost agent always incurs a higher loss than any other agent, while decreasing the others' average earnings. Second, by adding one link or replacing its current link with two others she always decreases own earnings and increases own envy by more than she decreases own guilt. She thus also becomes the lowest earning agent and her disadvantage only increases if she adds yet more links. Third, in treatments V and VV the central agent in PSS $_{o}^{-v}$ decreases own earnings, increases own guilt and has no envy by removing its only link. A direct calculation shows that also in treatment VV the central agent in $\mathrm{PSS}_{v}^{-v}$ does not want to remove its only link. Finally, a (normal-cost) central agent in $\mathrm{LS}_{c o}^{1}$ in treatment C, or in $\mathrm{LS}_{c v}^{1}$ or the remaining $\mathrm{LS}_{c v}^{2}$ in treatment $\mathrm{CV}$, when adding one or both her missing links decreases own earnings while the increase in own envy is not compensated by a sufficient decrease in own guilt.

Low cost agents face similar tradeoffs between their earnings and their inequity in their decisions to deviate from an equilibrium network. In particular, a periphery low-cost agent is not willing to add links, remove or move its only link, or replace it with two or more other links, as this decreases own earnings, increases own envy but does not sufficiently decrease own guilt. A low-cost agent, central in an equilibrium linked star is, for the same reason, neither willing to add new links nor move its links or replace them with others. Finally, a low-cost agent in the center of a star with five periphery players decreases her earnings as well as both her envy and guilt when she removes one or more of her links. A simple calculation shows that, when $\alpha_{c} \leqslant 4$ and when she sponsors no more than three links, she will not want to remove any. A similar calculation shows that she will not remove any of her links in an equilibrium linked star.

To conclude the proof we identify, for each network listed in the proposition, how an agent with a sufficiently high envy would deviate. In treatments $\mathrm{C}$ and $\mathrm{CV}$, the central agent in $\mathrm{CSS}_{c}$ or $\mathrm{MSS}_{c}$ would remove any single link when sponsoring more than three. In treatment $\mathrm{CV}$ the high-value agent in $\mathrm{LS}_{c v}^{2}$ would remove its link with the low-cost agent. In treatment VV, the normal agent in the center of $\mathrm{PSS}_{o}^{-v}$ would remove one link if she sponsors two. A normal agent in the (minor) center of $\mathrm{LS}_{o v}^{v}$ or $\mathrm{LS}_{o o}^{v-v}$ would remove the link with the periphery high-value agent.

\section{Appendix B. Instructions}

[Most of the text is the same for all treatments. Treatment-dependent text appears in italics and in brackets "[...]," where we indicate the treatments in which the text appears.]

Welcome to this experiment in decision-making. In this experiment you can earn money. The amount of money you earn depends on the decisions you and other participants make. Please read these instructions carefully. 
In the experiment you will earn points. At the end of the experiment we will convert the points you have earned into US dollars [in Europe: "euros"] according to the rate: $\mathbf{7 0}$ points equal \$1 [in Europe: "1 euro"]. You will be paid your earnings privately and confidentially after the experiment.

Throughout the experiment you are not allowed to communicate with other participants in any way. If you have a question please raise your hand. One of us will come to your desk to answer it.

\section{YOUR GROUP}

At the beginning of the experiment the computer will randomly assign you-and all other participants-to a group of 6 participants. Group compositions do not change during the experiment. Hence, you will be in the same group with the same people throughout the experiment. The composition of your group is anonymous. You will not get to know the identities of the other people in your group: neither during the experiment nor after the experiment. The other people in your group will also not get to know your identity. On your computer screen, you will be marked Me and each of the five other people in your group will be marked with a letter: A, B, C, D, and E. Each letter will correspond to the same participant in your group during the entire experiment.

\section{NUMBER OF ROUNDS}

The experiment consists of 30 rounds. You will receive 150 points at the beginning of the first round. In each round you can earn additional points. Your total earnings will be the sum of the initial 150 points and your earnings in each of the 30 rounds.

\section{YOUR LINKING DECISIONS (I)}

In each round you will make linking decisions. You will have the possibility to link with any other participant in your group. That is, you can make any number of links $(0,1,2,3,4$, or 5$)$. The link(s) you make in a particular round are only valid for that round.

As we explain below, you can be "directly linked," "indirectly linked" or "not linked" with any other member of your group. You are directly linked with another person if:

- you make a link to that person, or

- if that person makes a link to you, or

- if both of you link to each other.

Neighbors: The people in your group that you are directly linked with are called your neighbors.

You are indirectly linked with another person if that person is not your neighbor but there is a sequence of links between you and that person.

If there is no sequence of links between you and another person then you are not linked (neither directly nor indirectly) to that person.

Distance: The distance between you and someone you are linked with is the minimal number of links you have to count to go from you to that person. That is, the distance between you and one of your neighbors is always 1 . The distance between you and someone directly linked with one of your neighbors but not to you is 2 , and so on. Since there are five other people in your group, the distance between you and a person you are linked with is at least 1 and at most 5 .

\section{YOUR LINKING DECISIONS (II)}

For each (direct) link that you make you have to pay some costs. You also benefit from all your direct and indirect links with other persons. Other persons also benefit from all their direct and indirect links (including their links with you).

Colors: Each person in your group has a color. There are four green persons, one blue person, and one purple person. At the beginning of the experiment, the computer randomly chooses one person in your group to be blue, and another person to be purple. [In treatments $C$ and $C i$ these sentences explain that the group consists of "five green and one purple person"; in $V, V i$ : "five green and one blue"; in $V V$ : "four green and two blue"; in B: "six persons."] The colors of all people in your group remain the same during the entire experiment.

[Sentence in treatments $C, V, V V$, and $C V:$ Y You receive information about your color and the color of the other people in your group at the beginning of the experiment.

[Paragraph in treatments $\mathrm{Ci}, \mathrm{Vi}$, and $\mathrm{CVi}$ :] At the beginning of the experiment you will receive information about your own color. You will, however, receive no information about the colors of the other persons in your group. Throughout the experiment you will never see the colors of the other persons in your group. The other persons in your group will also receive only the information about their own color and will never see your color.

Costs of linking: In every round, each person incurs a cost for each link this person makes.

[List in treatments C, Ci, CV and CVi:] This cost depends on the color of the person:

- Each green ["or blue” added in CV and CVi] person incurs a cost of $\mathbf{2 4}$ points for each link this person makes.

- The purple person incurs a cost of $\mathbf{7}$ points for each link this person makes. 
[Two sentences in treatments $B, V, V V$, and Vi:] You incur a cost of $\mathbf{2 4}$ points for each link you make. Other persons also incur a cost of 24 points for each link they make.

These linking costs remain the same throughout the experiment. You do not incur any cost for the links that other persons make and other persons do not incur any cost for the links that you make.

Benefits of linking: You will earn points from being linked with other persons in your group. The amount you earn from being linked with another person depends on the distance between you and that person, and on the color of that person. Your benefits from your direct and indirect links are determined as follows:

For each ["green" added in $V, V V$, and $V i$; "green or purple" added in $C V$ and $C V i$ ] person you are linked with you receive

- 16 points if the distance between you and that person is 1 ,

- 12 points if the distance between you and that person is 2 ,

- 9 points if the distance between you and that person is 3 ,

- 7 points if the distance between you and that person is 4 ,

- 5 points if the distance between you and that person is 5 .

[List added in $V, V V, V i, C V$, and $C V i$ :] If you are linked with the blue person you receive

- 32 points if the distance between you and that person is 1 ,

- 24 points if the distance between you and that person is 2 ,

- 18 points if the distance between you and that person is 3 ,

- 14 points if the distance between you and that person is 4 ,

- 10 points if the distance between you and that person is 5 .

Your total benefits in points are therefore computed by summing the benefits you receive from all the persons you are linked with, directly or indirectly. The benefits for other persons in your group are calculated in the same way from their direct and indirect links.

Note. There is a possibility that you are (indirectly) linked with the same person in more than one way. However, you only benefit once from being linked to this person. Your benefits are determined by the shortest connection (the distance) between you and that person.

\section{CONCLUDING REMARKS}

You have reached the end of the instructions. It is important that you understand them. If anything is unclear to you or if you have questions, please raise your hand.

To ensure that you understood the instructions we ask you to answer a few control questions. After everyone has answered these control questions correctly the experiment will start.

\section{References}

Bala, V., Goyal, S., 2000. A non-cooperative model of network formation. Econometrica 68, 1131-1230.

Barabási, A.-L., 2002. Linked: The New Science of Networks. Promeus.

Barabási, A.-L., Albert, R., 1999. Emergence of scaling in random networks. Sci. 286 (286), 509-512.

Callander, S., Plott, C., 2005. Principles of network development and evolution: An experimental study. J. Public Econ. 89, 1469-1495.

Corbae, D., Duffy, J., 2008. Experiments with network formation. Games Econ. Behav. 64, 81-120.

Falk, A., Kosfeld, M., 2005. It's all about connections: Evidence on network formation. Working paper. University of Zürich.

Fehr, E., Schmidt, K.M., 1999. A theory of fairness, competition, and cooperation. Quart. J. Econ. 114, 817-868.

Freeman, L., 1979. Centrality in social networks: Conceptual clarification. Soc. Networks 1, 215-239.

Fudenberg, D., Tirole, J., 1991. Game Theory. MIT Press, Cambridge, MA.

Galeotti, A., Goyal, S., Kamphorst, J., 2006. Network formation with heterogeneous players. Games Econ. Behav. 54 (2), 353-372.

Goyal, S., van der Leij, M., Moraga-Gonzáles, J.L., 2006. Economics: An emerging small world. J. Polit. Economy 114 (2), 403-412.

Haller, H., Sarangi, S., 2005. Nash networks with heterogeneous links. Math. Soc. Sci. 50, 181-201.

Haller, H., Kamphorst, J., Sarangi, S., 2007. (Non-)existence and scope of Nash networks. Econ. Theory 31 (3), 597-604.

Jackson, M.O., 2004. A survey of models of network formation: Stability and efficiency. In: Demange, G., Wooders, M. (Eds.), Group Formation in Economics:

Networks, Clubs and Coalitions. Cambridge University Press, Cambridge.

Jackson, M.O., 2005. The economics of social networks. In: Blundell, R., Newey, W., Persson, T. (Eds.), Proceedings of the 9th World Congress of the Econometric Society. Cambridge University Press, Cambridge.

Jackson, M.O., Rogers, B., 2007. Meeting strangers and friends of friends: How random are socially generated networks? Amer. Econ. Rev. 97 (3), $890-915$. Jackson, M.O., Wolinsky, A., 1996. A strategic model of social and economic networks. J. Econ. Theory 71, 44-74.

Johnson, C., Gilles, R.P., 2000. Spatial social networks. Rev. Econ. Design 5, 273-299.

Kosfeld, M., 2004. Economic networks in the laboratory: A survey. Rev. Network Econ. 3, 20-42.

McBride, M., 2008. Position-specific information in social networks: Are you connected? Math. Soc. Sci. 56, 283-295.

Newman, M.E.J., 2004. Coautorship networks and patterns of scientific collaboration. Proceed. Nat. Acad. Sci. 101, 5200-5205.

Wasserman, S., Faust, K., 1994. Social Network Analysis: Methods and Applications. Cambridge University Press. 